\title{
Scattering of Stationary and Non-Stationary Sound by Ideal and Elastic Scatterers, Placed near Interface of Media, in Underwater Sound Channel and in Plane Waveguide
}

\author{
Alexander Kleshchev \\ Department of Physics, Saint-Petersburg State Technical University, Saint-Petersburg, Russia \\ Email: Alexalex-2@yandex.ru
}

How to cite this paper: Kleshchev, A. (2017) Scattering of Stationary and NonStationary Sound by Ideal and Elastic Scatterers, Placed near Interface of Media, in Underwater Sound Channel and in Plane Waveguide. Open Access Library Journal, 4: e4141.

https://doi.org/10.4236/oalib.1104141

Received: November 10, 2017

Accepted: December 12, 2017

Published: December 15, 2017

Copyright $\odot 2017$ by author and Open Access Library Inc.

This work is licensed under the Creative Commons Attribution International License (CC BY 4.0).

http://creativecommons.org/licenses/by/4.0/

\begin{abstract}
In the review we obtained a strict solution of problem of the sound diffraction by an elastic spheroidal shell, located near the interface of a liquid medium with an elastic solid medium. Calculation of the scattered sound field for ideal bodies (a spheroid and an elliptical cylinder) is performed. These bodies are placed on the interface between the liquid and the ideal medium; it is shown, that the main role is played not by interaction of scatterers (real and imaginary), but by interference of their scattered fields. The spectrum of the scattered impulse signal for the body in an underwater sound channel is calculated. It is shown, that at large distances the dominante role is played by the spectral characteristic of the channel itself. Based on the method of imaginary sources and imaginary scatterers, the solution of the current study is to solve the diffraction problem of sound pulse signals at ideal (soft) prolate spheroid, which is put in the plane waveguide with the hard elastic bottom. In the work, it is proved that with such a formulation of problems eliminated, there exists possibility of using the method of normal waves because pulses are bundies of energy and can therefore only be distributed to the group velocity which is inherent in just the method of imaginary sources. Calculations made in the article showed that imaginary sources with small numbers exert the effect of total internal reflection, as the result of the reflection coefficient $V$ by the hard elastic bottom which is complex and the real part of $V$ is close to 1.0 which corresponds to $V$ absolutely hard bottom. Found sequences of reflected pulses for the elastic hard bottom and the absolutely hard bottom floor confirmed this approach. In the final part of the article, on the basis of the received results, a solution (the method integral equations) is given, which is a much more complex problem of the diffraction at the elastic non-analytical scatter-
\end{abstract}


er, put in the plane waveguide with the hard elastic bottom.

\section{Subject Areas}

Applied Physics, Experimental Physics

\section{Keywords}

Scatterer, Prolate Spheroid, Imaginary Source, Diffraction, Elastic Hard

Bottom, Boundary Conditions, Group Velocity, Phase Velocity, Underwater

Sound Channel, Plane Waveguide

\section{Introduction}

In the series of problems on the study of the influence of media interfaces on characteristics of sound scattering by bodies, following variants are usually investigated:

a) An interaction of a scatterer with a single interface between media;

b) The scatterer in the field of interfracting modes of the underwater sound channel;

c) The finding of the total scattered field of the system of real and imaginary sources and scatterers of the plane waveguide.

\section{Elastic Scatterer near Interface of Media}

The interaction of the scatterer with the interface between media is considered in the example of the problem of scattering of sound by an elastic spheroidal body located at the interface between a liquid an elastic medium [1]. Let the elastic gas-filled prolate spheroidal shell be placed near the boundary a liquidan elastic medium (Figure 1). The axis of rotation of the shell is parallel to the plane boundary. We introduce two systems of spheroidal coordinates $\xi_{s}, \eta_{s}, \varphi_{s}(s$ $=1,2)$, the first of which $(s=1)$ we associate with the scatterer, the second $(s=2)$ with the interface plane. The beginning of the Cartesian coordinate system $\mathrm{O}_{2}$ foci of the second spheroidal coordinate system are defined as projections respectively $O_{1}$ and foci of the first coordinate system on the plane of the boundary $Z_{2} Y_{2}$, so that inter-focus distance $2 h_{0}$ is common for both coordinate systems. The interface plane is two coordinate half-planes $\left(\varphi_{2}^{\prime}=\pi / 2\right.$ and $\left.\varphi_{2}^{\prime \prime}=-\pi / 2\right)$ coordinate system. In order to relate this solution to the solution of the diffraction problem on the elastic spheroidal shell, we simplify the formulation of the problem and assume that the wave vector $\boldsymbol{k}$ of the incident wave is in the plane $X_{1} Z_{1}$ (and correspondly $X_{2} Z_{2}$ ), $\varphi_{\text {os }}=0^{\circ} \quad$ (see Figure 1 ).

Now along with the potential $\Phi_{1}^{(1)}$ of the wave, scattered by the shell, will appear the potential $\Phi_{1}^{(2)}$ frothe elastic half-space:

$$
\Phi_{1}^{(2)}=2 \sum_{m=0}^{\infty} \sum_{n \geq m}^{\infty} K_{m, n} \bar{S}_{m, n}\left(C_{1}, \eta_{2}\right) R_{m, n}^{(1)}\left(C_{1}, \xi_{2}\right) \cos m \varphi_{2} .
$$




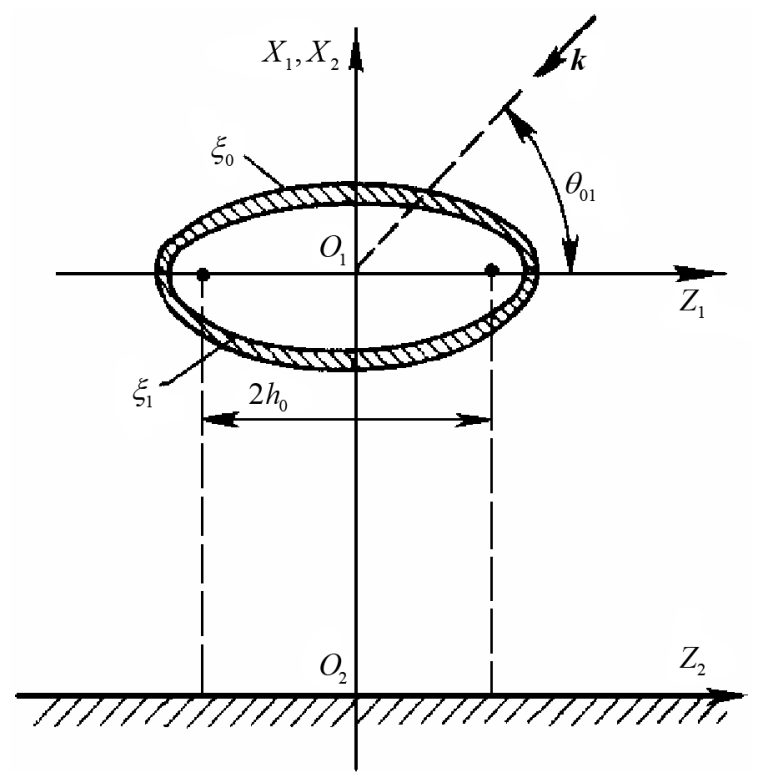

Figure 1. Elastic spheroidal shell near the liquid-elastic isotropic medium interface.

The potential $\Phi_{1}^{(2)}$ decomposes by radial functions of the first kind, this is due to the fact, that foci of the second coordinate system lie in the plane of the interface, physically this means that the interacting of scatterers are distorted by fields of two plane waves: an incident and reflected by an interface. Expansions of potentials of the shell are accompanied by expansions of potentials of an elastic half-space:

$$
\begin{gathered}
U_{2}^{(2)}=2 \sum_{m=1}^{\infty} \sum_{n \geq m}^{\infty} M_{m, n} \bar{S}_{m, n}\left(C_{t_{2}}, \eta_{2}\right) R_{m, n}^{(1)}\left(C_{t_{2}}, \xi_{2}\right) \sin m \varphi_{2} ; \\
W_{2}^{(2)}=2 \sum_{m=0}^{\infty} \sum_{n \geq m}^{\infty} N_{m, n} \bar{S}_{m, n}\left(C_{t_{2}}, \eta_{2}\right) R_{m, n}^{(1)}\left(C_{t_{2}}, \xi_{2}\right) \cos m \varphi_{2}, \\
\Phi_{2}^{(2)}=2 \sum_{m=0}^{\infty} \sum_{n \geq m}^{\infty} L_{m, n} \bar{S}_{m, n}\left(C_{l_{2}}, \eta_{2}\right) R_{m, n}^{(1)}\left(C_{l_{2}}, \xi_{2}\right) \cos m \varphi_{2} ;
\end{gathered}
$$

where $C_{l_{2}}$ and $C_{t_{2}}$-are wave dimensions of longitudinal transverse waves respectively.

The potential of the incident wave $\Phi_{0}$ in two coordinate systems has a form [2] [3]:

$$
\Phi_{0}=2 \sum_{m=0}^{\infty} \sum_{n \geq m}^{\infty} i^{-n} \varepsilon_{m} \bar{S}_{m, n}\left(C_{1}, \eta_{0}\right) \bar{S}_{m, n}\left(C_{1}, \eta_{s}\right) R_{m, n}^{(1)}\left(C_{1}, \xi_{s}\right) \cos m \varphi_{s},(s=1,2) .
$$

The potential of a diffracted field $\Phi_{\Sigma}=\Phi_{0}+\Phi_{1}^{(1)}+\Phi_{2}^{(2)}$ obeys simultaneously to boundary conditions on a surface of a shell and on a planar interface between a liquid and an elastic medium. To boundary conditions on a surface of a shell are supplemented by conditions on an inter-face between a liquid-elastic medium:

$$
\lambda_{0} k^{2}\left(\Phi_{0}+\Phi_{1}^{(1)}+\Phi_{1}^{(2)}\right)=\lambda_{2} k_{l_{2}}^{2} \Phi_{2}^{(2)}+\left.2 \mu_{2} u_{\varphi \varphi}^{(2)}\right|_{\varphi=\pi / 2 ;-\pi / 2} ;
$$




$$
\begin{aligned}
& \left(h_{\varphi}^{(2)} / h_{\xi}^{(2)}\right)(\partial / \partial \xi)\left(u_{\varphi}^{(2)} / h_{\varphi}^{(2)}\right)+\left.\left(h_{\xi}^{(2)} / h_{\varphi}^{(2)}\right)(\partial / \partial \varphi)\left(u_{\xi}^{(2)} / h_{\xi}^{(2)}\right)\right|_{\varphi=\pi / 2 ; \pi / 2}=0 \\
& \left(h_{\varphi}^{(2)} / h_{\xi}^{(2)}\right)(\partial / \partial \xi)\left(u_{\varphi}^{(2)} / h_{\varphi}^{(2)}\right)+\left.\left(h_{\xi}^{(2)} / h_{\varphi}^{(2)}\right)(\partial / \partial \varphi)\left(u_{\xi}^{(2)} / h_{\xi}^{(2)}\right)\right|_{\varphi=\pi / 2 ;-\pi / 2}=0 ; \\
& -\left(h_{\varphi}\right)^{-1}(\partial / \partial \varphi)\left(\Phi_{0}+\Phi_{1}^{(1)}+\Phi_{1}^{(2)}\right)=\left(h_{\varphi}^{(2)}\right)^{-1}\left(\partial \Phi_{2} / \partial \varphi\right) \\
& +\left.\left(h_{\xi}^{(2)} h_{\eta}^{(2)}\right)^{-1}\left[(\partial / \partial \xi)\left(h_{\eta}^{(2)} \psi_{\eta}^{(2)}\right)-(\partial / \partial \eta)\left(h_{\xi}^{(2)} \psi_{\xi}^{(2)}\right)\right]\right|_{\varphi=\pi / 2 ;-\pi / 2},
\end{aligned}
$$

where $\lambda_{2}$ and $\mu_{2}$-Lame coefficients of an elastic half-space; $k_{l}^{(2)}$-a wavt number of a longitudinal wave of an elastic half-space.

In our formulation of a problem because of a panty of a solution respect a plane $X Z$ boundary conditions for $\varphi=-\pi / 2$ completely repeat conditions for $\varphi=+\pi / 2$, that do not provide any additional information in this case. When substituting potential expansions into boundary conditions for a shell and a planar interface when an expansion coefficients is used an addition theorem for wave spheroidal functions [3],

$$
\begin{aligned}
& R_{p, q}^{(1),(3)}\left(C_{j}, \xi_{j}\right) \bar{S}_{p, q}\left(C_{j}, \eta_{j}\right) \exp \left(i p \varphi_{j}\right) \\
& =\sum_{n=0}^{\infty} \sum_{m=-n}^{n} R_{m, n}^{(1)}\left(C_{s}, \xi_{s}\right) \times \bar{Q}_{m, n, p, q}^{(1),(3)}\left(C_{j}, C_{s} ; l ; \theta_{j s}\right) \bar{S}_{m, n}\left(C_{s}, \eta_{s}\right) \exp \left(i p \varphi_{s}\right),
\end{aligned}
$$

where

$$
\begin{aligned}
\bar{Q}_{m, n, p, q}^{(1),(3)}= & 2(-i)^{n-q} \sum_{r=0,1}^{\infty} \sum_{t=0,1}^{\infty}{ }^{\prime} d_{r}^{p q}\left(C_{j}\right) d_{t}^{m n}\left(C_{s}\right) \sum_{\sigma=|r+p-t-m|}^{r+p+t+m}(-i)^{\sigma} \\
& \times \tilde{b}_{\sigma}^{(r+p, p, t+m, m)} Z_{\sigma}^{(1),(3)}(k l) \tilde{P}_{\sigma}^{p-m}\left(\theta_{j s}\right) \exp \left[i(p-m) \varphi_{j s}\right] ;
\end{aligned}
$$

$\theta_{j s}$ - polar angle of a point $O_{s}$-a beginning of a $s$-th coordinate system in an $i$-th system (Figure 2); 1 -a distance between $O_{j}$ and $O_{s} \quad d_{r}^{p q}\left(C_{j}\right)$ and $d_{t}^{m n}\left(C_{s}\right)$ -coefficients of expansions $\mathrm{f}$ functions $\bar{S}_{p, q}\left(C_{j}, \eta_{j}\right)$ and $\bar{S}_{m, n}\left(C_{s}, \eta_{s}\right)$ by functions $\tilde{P}_{r}^{p}\left(\eta_{j}\right)$ and $\tilde{P}_{t}^{m}\left(\eta_{s}\right)$, which up to a constant factor coincide with normalized adjoint Legendre functions; $Z_{\sigma}^{(1)}=j_{\sigma}(k l)$-spherical Bessel functions; $Z_{\sigma}^{(3)}=h_{\sigma}^{(1)}(k l)$-spherical Hanrtl functions of a first kind; coefficients $\tilde{b}_{\sigma}^{(r+p, p, t+m, m)}$ are obtained from coefficients $b_{\sigma}^{(r+p, p, t+m, m)}$ [3] taking into account a relationship $\tilde{P}_{t}^{m}\left(\eta_{s}\right)$ and associated Legendre functions; a prime for signs $\Sigma$ means that a summation is carried out on an ever basis $r$ and $t$, if, respectively, $g-p$ and $n-m$ is even and $r$ and $t$ is odd, if $g-p$ and $n-m$ is odd.

A strict solution can be obtained for another orientation of a spheroidal shell with respect to a plane bourder, namely, under a condition of perpendicularity of a rotation axis of a shell to a plane interface between media (Figure 3). We will consider this orientation in more detail, by replacing a spheroidal shell with an ideal prolate soft spheroid and an elastic half-space by an ideal medium (hard or soft) [4]. We will map a scatterer and a source mirror-wise with respect to a boundary and reduce a problem to diffraction of fields of two sources (real and imaginary) by two spheroidal scatterers (real and imaginary). Potentials $\Phi_{s}(S=$ 1,2 ) of waves scattered by spheroids are chosen in a form of expansions (taking into account an axial symmetry) [4]: 


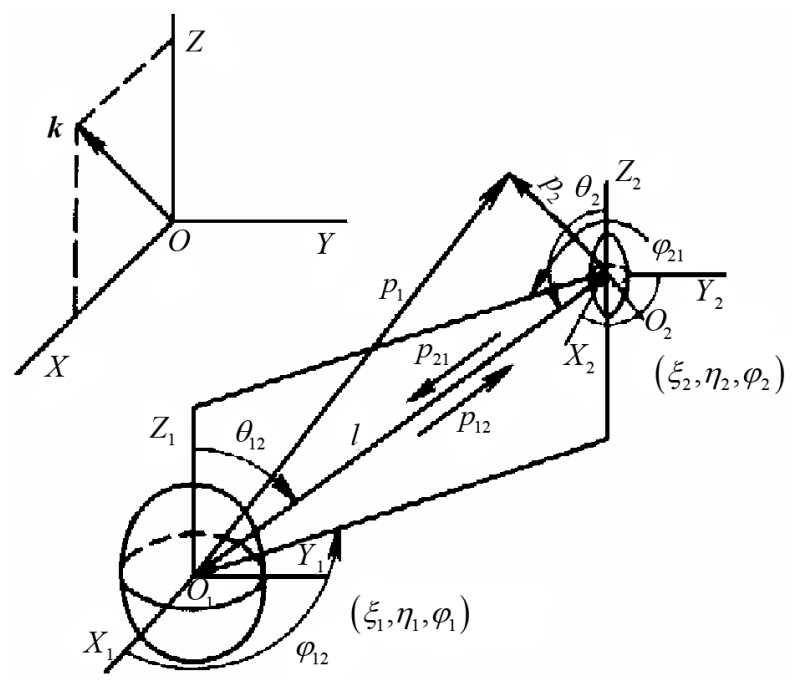

Figure 2. Systems of spheroidal coordinates associated interface with two interacting scatterers.

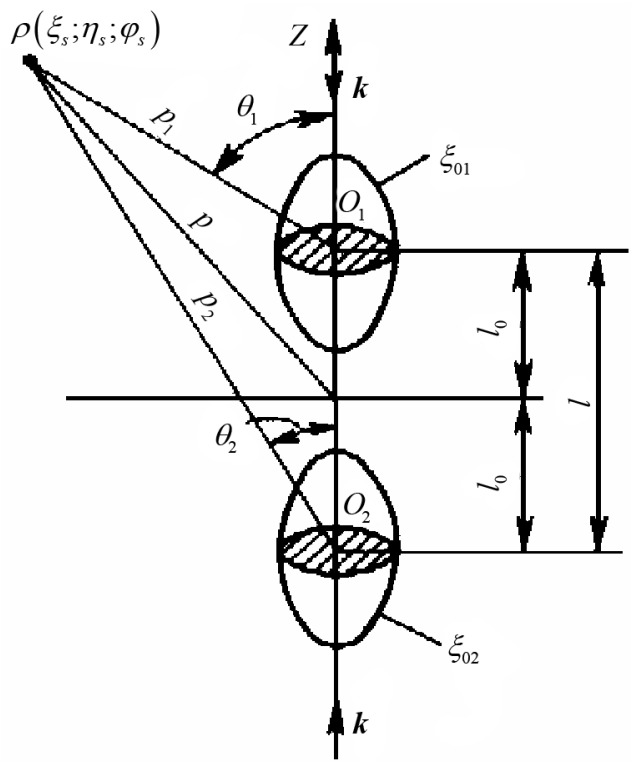

Figure 3. Ideal spheroid at an face.

$$
\Phi_{s}\left(\xi_{s}, \eta_{s}\right)=\sum_{n=0}^{\infty} B_{0, n}^{s} \bar{S}_{0, n}\left(C_{s}, \eta_{s}\right) R_{0, n}^{(3)}\left(C_{s}, \xi_{s}\right) .
$$

Since spheroids (real and imaginary) are ideally soft, then on their surfaces $\left(\xi_{0}=\xi_{01}=\xi_{02}\right)$ is satisfied a homogeneous Dirichlet:

$$
\Phi_{0}+\sum_{s=1}^{2} \Phi_{s}=\left.0\right|_{\xi=\xi_{0} ; s=1,2} .
$$

A potential of a falling plane wave is given by a decomposition:

$$
\Phi_{0}\left(\xi_{s}, \eta_{s}\right)=2 \sum_{n=0}^{\infty} i^{-n} \bar{S}_{0, n}\left(C_{s}, \eta_{s}\right) R_{0, n}^{(1)}\left(C_{s}, \xi_{s}\right) \bar{S}_{0, n}\left(C_{s}, 1\right), s=1,2 .
$$

Unknown coefficients $B_{0, n}^{s}$ of expansions (11) are sought from an infinite 
system of equations-boundary conditions (12) [4]:

$$
\begin{aligned}
& B_{0, n}^{s}+\sum_{q=0}^{\infty} B_{0, q}^{t} R_{0, n}^{(1)}\left(C_{s}, \xi_{0 s}\right)\left[R_{0, n}^{(3)}\left(C_{s}, \xi_{0 s}\right)\right]^{-1} \bar{Q}_{0 n 0 q}^{(3)}\left(C_{t}, C_{s} ; l ; \theta_{t s}\right) \\
& =-2 i^{-n} \bar{S}_{0 n}\left(C_{s}, 1\right) R_{0, n}^{(1)}\left(C_{s}, \xi_{0 s}\right)\left[R_{0, n}^{(3)}\left(C_{s}, \xi_{0 s}\right)\right]^{-1}, s=1,2 ; t=1,2 ; s \neq t,
\end{aligned}
$$

where 1 -a distance between centers of coordinate systems $O_{1}$ and $O_{2}$ (Figure 3), in our case:

$$
\begin{aligned}
& \theta_{12}=0, \theta_{21}=\pi \\
& \bar{Q}_{0 n 0 q}^{(3)}\left(C_{t}, C_{s} ; l ; \theta_{t s}\right) \\
& =2 i^{-n+q} \sum_{r=0,1}^{\infty} \sum_{j=0,1}^{\infty} ' d_{r}^{0 q}\left(C_{t}\right) d_{j}^{0 n}\left(C_{s}\right) \sum_{\sigma=|r-j|}^{r+j} i^{-\sigma} b_{\sigma}^{(r, 0, j, 0)} h_{0}^{(1)}(k l) P_{\sigma}\left(\cos \theta_{t s}\right) ; \\
& b_{\sigma}^{(r, 0, j, 0)}=(r j 00 \mid \sigma O)^{2} .
\end{aligned}
$$

For a regularization of a system (14) with respect to unknown coefficients $B_{0, n}^{s} \quad$ we introduce new unknown $X_{0, n}^{s}$ from a ratio [4]:

$$
B_{0, n}^{s}=R_{0, n}^{(1)}\left(C_{s}, \xi_{0 s}\right) X_{0, n}^{s} .
$$

As a result, an infinite system (14) for unknown $B_{0, n}^{s}$ is reduced to another infinite system of relatively new unknown $X_{0, n}^{s} \quad[4]$ :

$$
\begin{aligned}
& X_{0, n}^{s}+\sum_{q=0}^{\infty} X_{0, q}^{t} R_{0, q}^{(1)}\left(C_{t}, \xi_{0 t}\right)\left[R_{0, n}^{(3)}\left(C_{s}, \xi_{0 s}\right)\right]^{-1} \bar{Q}_{0 n 0 q}^{(3)}\left(C_{t}, C_{s} ; l ; \theta_{t s}\right) \\
& =-2 i^{-n} \bar{S}_{0, n}\left(C_{s}, 1\right)\left[R_{0, n}^{(3)}\left(C_{s}, \xi_{0 s}\right)\right]^{-1} .
\end{aligned}
$$

Further we find by a trunkaction method a solution of a regular system (16). Initially we calculated angular scattering functions $D_{s}\left(\theta_{s}\right)$ of two interacting spheroids distorting a field of a plane monochromatic wave. A Figure 4 shows

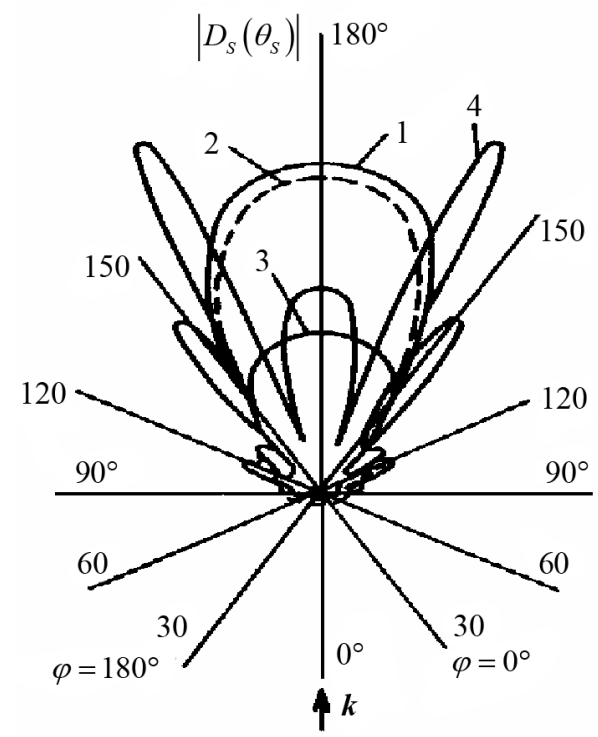

Figure 4. Modules of angular characteristics of single and interacting spheroids. 
moduluses of angular characteristics $\left|D_{s}\left(\theta_{s}\right)\right|$ of tnteracting spheroids (a curve 1 refers to a first spheroid, a curve 2-to a second spheroid). A curve 3 depicts in another scale $|D(\theta)|$ of a single soft spheroid in an infinite medium. A scale had to be changed so that a curve 3 did not merge with curves 1 and 2. A curve 4 characterizes a modulus $\left|D_{\Sigma}\left(\theta_{1}\right)\right|$ of a total angular characteristic in coordinates of a first spheroid (Figure 1):

$$
\left|D_{\Sigma}\left(\theta_{1}\right)\right|=\left|D_{1}\left(\theta_{1}\right)+D_{2}\left(\theta_{1}\right) \exp \left(i k l \cos \theta_{1}\right)\right| .
$$

Calculations were carried out at: $C_{1}=C_{2}=10.0, \xi_{01}=\xi_{02}=\xi_{0}=1.005$, a semi-focus distance $h_{01}=h_{02}=1 \mathrm{M}, l=8 h_{0}$. An analysis of curves prestnted in Figure 4 shows that when selected parameters $\left(C_{1}, C_{2}, I\right)$ an interaction of scatterers turned out to be smail, because of this curves $1,2,3$ are so close to each other. A main role is played by interference effects (especially in a shadow region), so a curve 4 stands out sharply (again in a shadow region) agatnst a background of other curves. In a second stage (based on a calculation of a scattered field of two spheroids) were calculated angular characteristics $D_{\Sigma}(\theta)$ of a soft spheroid $\left(\xi_{0}=1.005 ; C=10\right)$, located at a distance $l_{0}=4 h_{0}=4 \mathrm{~m}$ from an interface between liquid and an ideal medium. Results of calculations $\left|D_{\Sigma}(\theta)\right|$ are shown in Figure 5. A curve 1 corresponds to an interface between liquid and soft media. A certain contribution to $D_{\Sigma}(\theta)$ is made by an imaginary source and associated with it interference fringes of both spheroids, physically intense scattering in an illuminated region anses because of a reflection of waves scattered by spheroids by a plane interface between media. We must not forget that I this case we are not talking about a distortion of a plane traveling wave (as it was an unlimited medium), about a distortion of a standing wave field real and imaginary sources.

A strict solution also has a problem of scattering of sound by a spheroidal half-body placed on an interface between a liquid and an ideal medium. With a mirror image of a scatterer and a source with respect to a plane boundary, we obtain a spheroidal scatterer located in a field of two sources (real and imaginary).

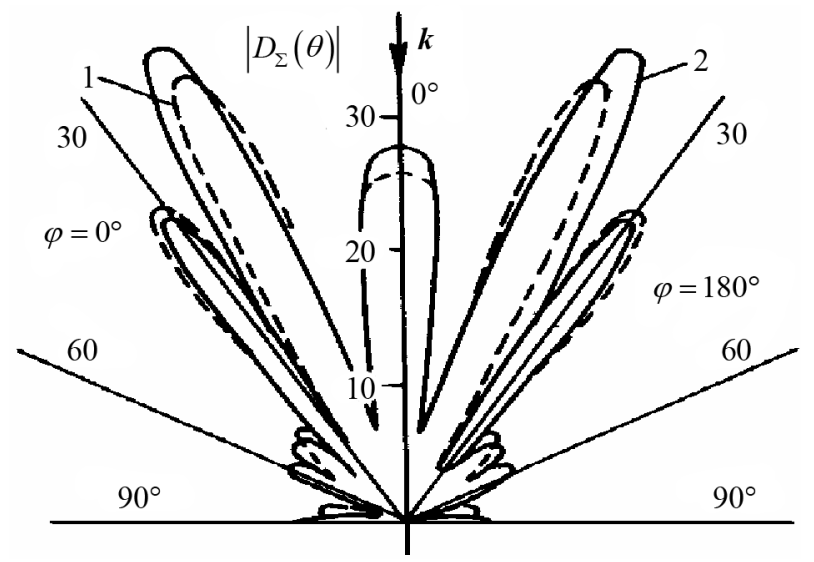

Figure 5. Modules of angular characteristics of soft spheroid at an interface of media. 
A phase of a wave potential from an imaginary source on a plane interface coincides with a phase of a potential of an incident wave in this plane in a liquid borders on an ideally rigid medium and phases of these waves differ at a boundary by $180^{\circ}$ if a liquid borders on an ideally soft medium. In Figure 6 shows modules of angular characteristics $|D(\theta)|$ (in .different scales) of spheroidal half-bodies located on a boundary of a liquid with an ideal medium. A curve 1 corresponds to half a hollow steel oblate spheroidal shell placed on a boundary of a liquid an ideally soft medium. Outher radial coordinate of a shell $\xi_{0}=0.1005$, internal $-\xi_{1}=0.07669$, a wave size $C=7.1$, an irradiation angle $\theta_{0}=0^{\circ}$ (an axisymmetric problem). Two other curves (see Figure 6) relate to modulus of angular characteristics of a soft oblate hemispheroid bordering on soft (a curve 2) and hard (a curve 3) media at a same irradiation angle $\theta_{0}=0^{\circ}$ and a wave size of a body $C=10.0$, a radial coordinate of a soft oblate spheroid $\xi_{0}=0.1005$.

In Figure 7 show relative cross sections for backscattering $\sigma_{0}$ of oblate ideal hemispheroids located on interface between media, where n under $D(\theta ; \varphi)$ we mean a total angular characteristic (from real and iimaginary sources). Curves of Figure 7 correspond to axially radiation $\left(\theta_{0}=0^{\circ}\right)$ of an oblate hemispheroid (soft or hard) with a radial coordinate $\xi_{0}=0.1005$. Curves 1 and 3 refer to a hard hemispheroid placed at a boundary with a hard 1 and soft 3 media (a liquid we have designated by a number I, soft-by a number II, hard-a number III). Curves 3 and 4 correspond to a soft hemispheroid located at a boundary with soft 2 and hard 4 media. Curves 5 and 6 give an idea of a variation $\sigma_{0}$ of hard 5 and soft 6 oblate spheroids $\left(\xi_{0}=0.1005\right.$ ) in an infinite liquid medium. It is easy to see that if a hemispheroid and an ideal semibounded medium and same (hard

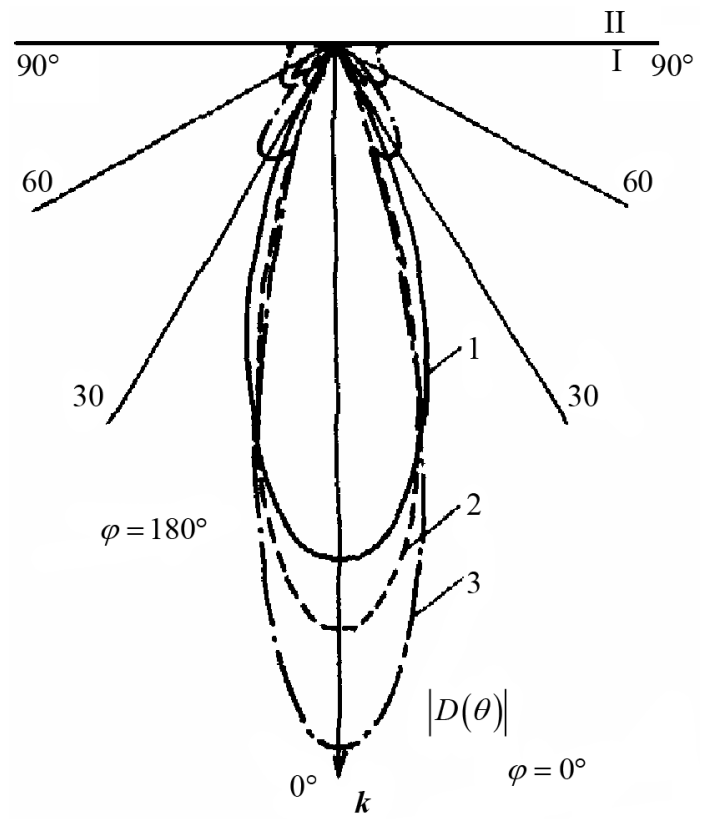

Figure 6. Modules of angular characteristics of spheroidal semi-bodies located an interface between media. 


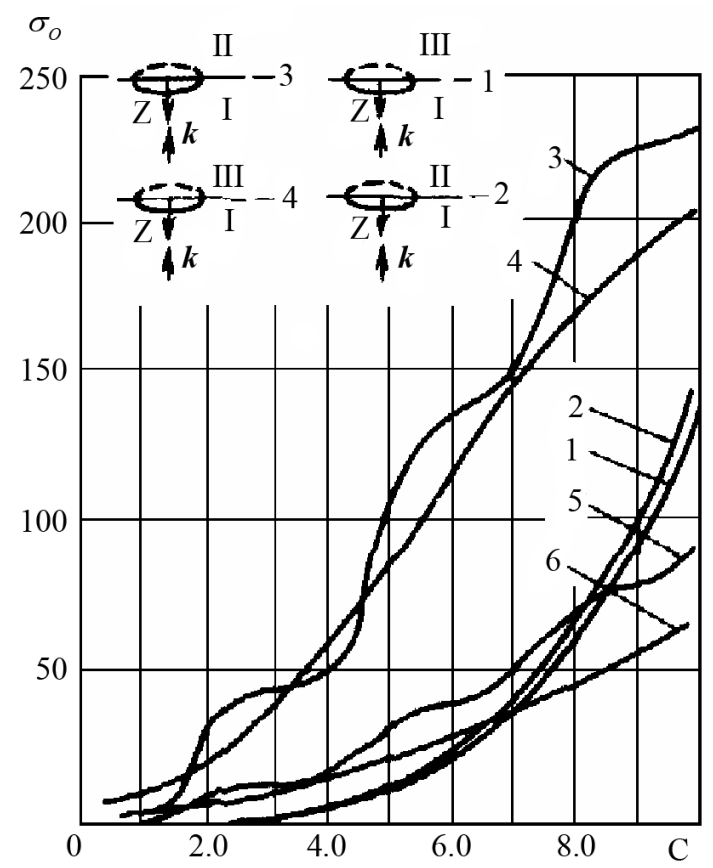

Figure 7. Relative cross sections for back-scattering of oblate spheroids.

or soft, curves 1 and 2) that is, a half body represents only a violation of a shape of a boundary hides this unevenness and $\sigma_{0}$ grows slowly and at smail wave dimensions close to zero and vise versa, for different materials of a hemispheroid and a semibouded ideal medium cross-sections $\sigma_{0}$ are much larger (curves 3 and 4 ) in an entre investigated range of wave dimensions. While acurves 5 and 6 tend asymptotically to a value of geometric acoustics, remaining curves increase indefinitely. Mathematical and physical explanations for this phenomenon were given in comments to Figure 5.

Let us turn to passive characteristics (scattering indicatrix) of hemispheroids located on an interface between a liquid and an ideal medium. We imagine for this that on a fairly large distance from a boundary along a line $L M$ a combined system (source-receiver) moves, while we are interested in a reflected signal to a point finding a combined system. A movement of a system is so slow that a Doppler effect can be ignored. Two orientations of half-bodies are possible which admit are depicted in Figure 8 and Figure 9. In Figure 10 (in different scales) presented modules $\left|D_{\Sigma}^{0}(\theta)\right|$ for a prolate soft hemispheroid on a boundary of a liquid with an ideally hard medium (a curve 1, its scale is shown to a left of a vertical axis, $\xi_{0}=1.005, C=10$ ) and for an oblate hard hemispheroid on a boundary of a liquid with an ideally soft medium (a curve 2, its scale is shown to a right of a vertical axis, $\xi_{0}=0.1005 ; C=10$ ). A orientation of hemibodies corresponds to Figure 8, directions of rays-by a oblate coordinate system. In Figure 11 shows modules $\left|D_{\Sigma}^{0}(\theta)\right|$ for a prolate hard hemispheroid on a boundary of a liquid with a ideally soft medium (a curve $1, \xi_{0}=1.005$, $C=10$ ) and for a oblate hard hemispheroid on a boundary of a liquid with a 


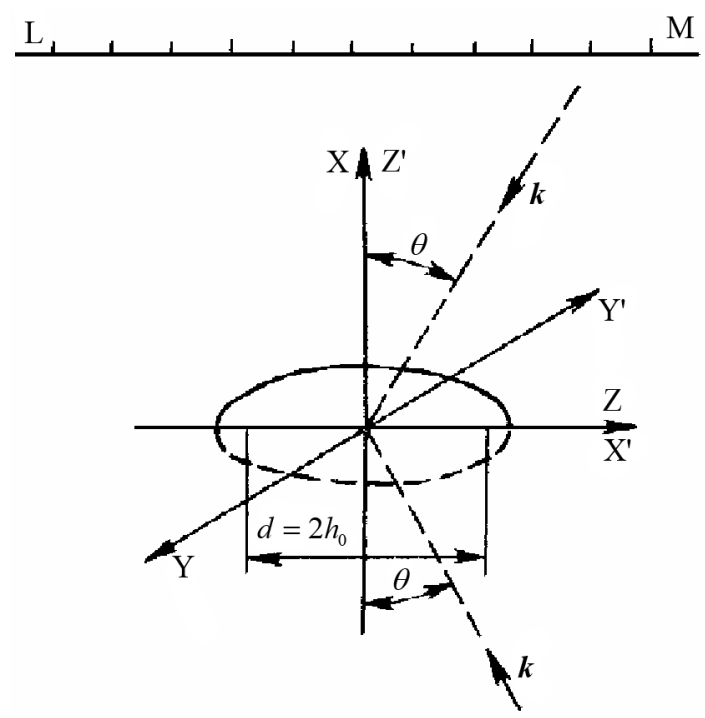

Figure 8. The multual orientation of a combined system and hemispheroid (1-st variant).

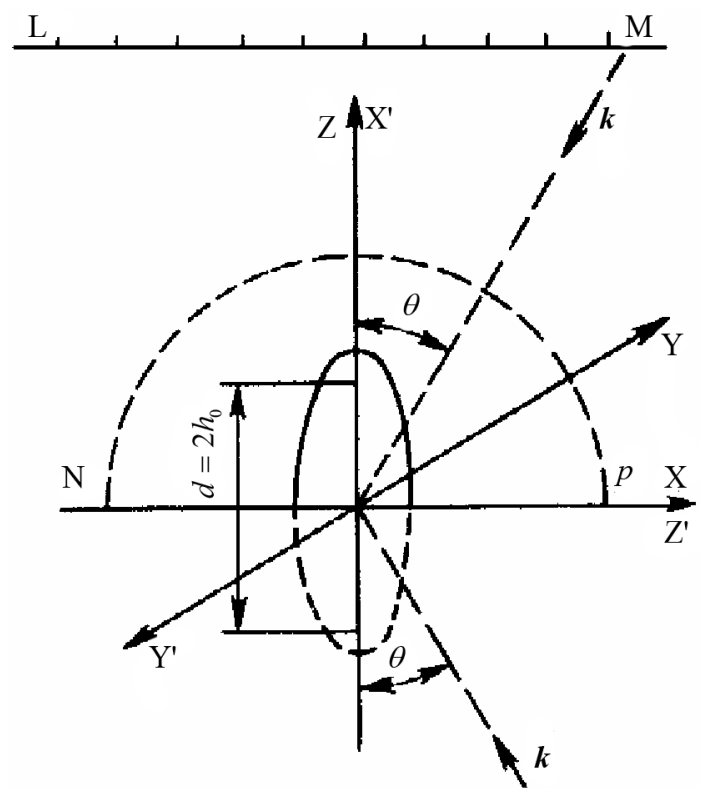

Figure 9. The mutual orientation of a combined system and hemispheroid (2-nd variant).

ideally sofy medium (a curve $2, \xi_{0}=0.1005 ; C=10$ ). A orientation of hemibodies corresponds to Figure 9, directions of rays-by a prolate coordinate system. An analysis presented scattering indicatrix shows an advantage of a second type orientation (see Figure 9), since in this case appears an intense non-mirror component, associated with sound reflection of an interface. At orientation of 1-st type (see Figure 8), intensive back reflection at $\theta=0^{\circ}$ will be masked by reflection from a planar interface.

We will pass from stationary (harmonic) irradiation to nonstationary radiation in a form of sound pulses with a rectangular envelope and monochromatic 


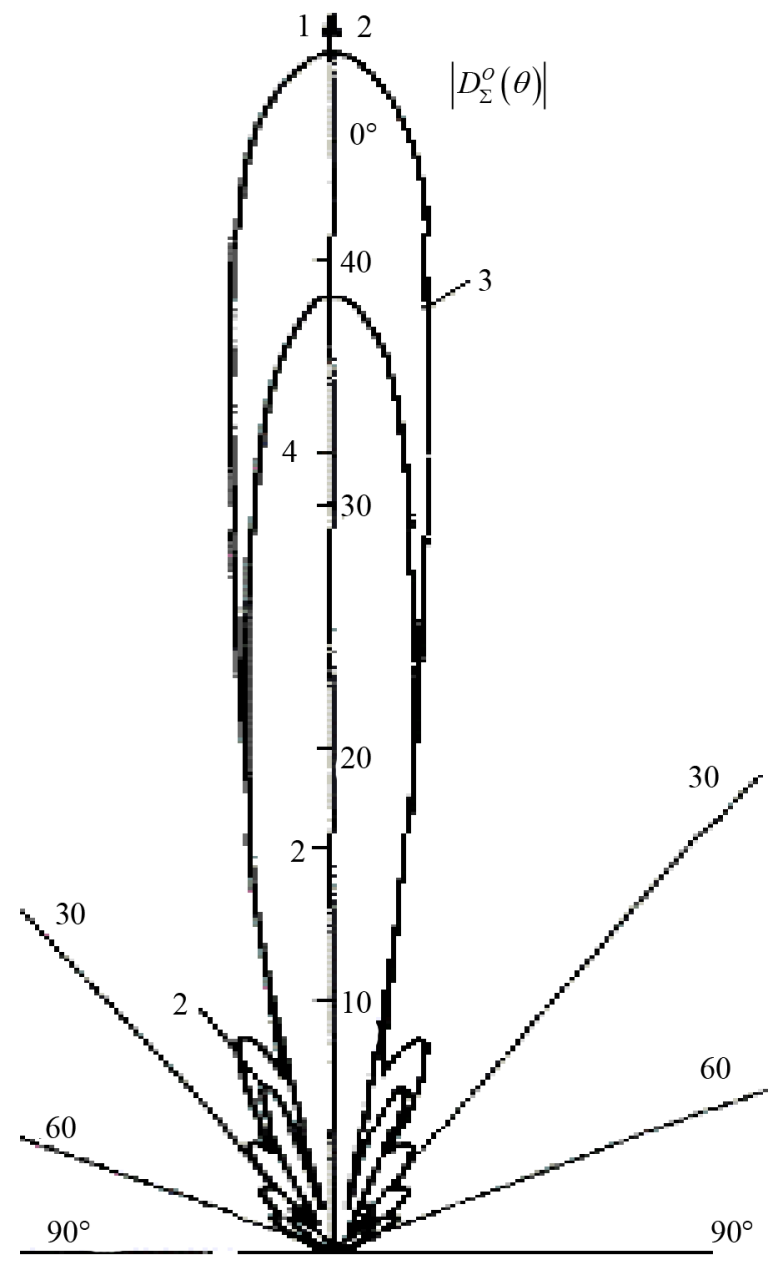

Figure 10. Modules of scattering indicatrics of soft hemispheroids.

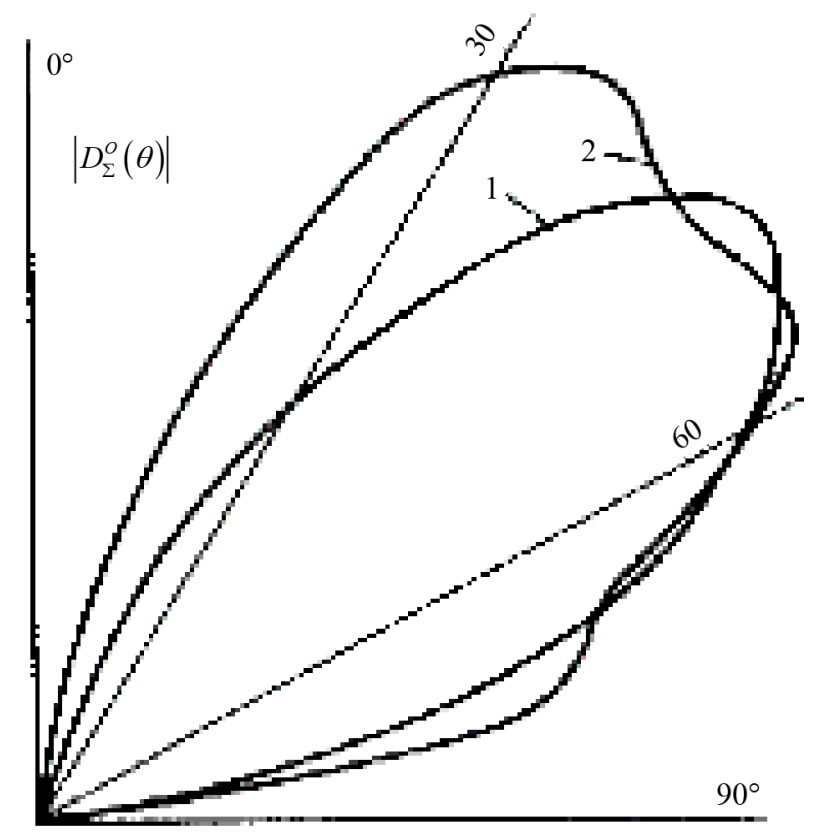

Figure 11. Modules of scattering indicatrics of hard hemispheroids. 
filling. As before, we will consider interfaces of media of three types: a liquid —an elastic bottom; a liquid—an ideally soft medium (Dirichlet condition); a liquid - an ideally hard medium (Neumann condition).

If a scatterer (oblate hard spheroid) is placed at an interface between media (a liquid-an elastic bottom) at an observation point, come first an impulse of a mirror reflection from a scatterer.

Figure 12, a shows a mirror reflection pulse $r_{1} \cdot \Psi_{S}(t)$ for a hard oblate spheroid when it is irradiated at an angle $\theta_{1}=30^{\circ}$; a normalized modulus of a spectrum of a pulse $r_{1} \cdot \Psi_{S}(t)$ is seen from Figure 12(b). After a while an observation point $P$ will reach an pulse $\Psi_{\Sigma}\left(t^{\prime}\right)$ reflected from an elastic bottom and diffracted on a spheroid.

Figure 13, a shows a pulse $\Psi_{\Sigma}(t)$, a normalized modulus spectrum $\left|S_{\Sigma}(v)\right|$ of a pulse $\Psi_{\Sigma}\left(t^{\prime}\right)$, presented on a Figure 13(b).

We orient a hard prolate hemispheroid in such a way that it's a major semiaxis will be in a plane of an interface between media. We calculate mirror-reflected pulses $\Psi_{\Sigma}\left(t^{\prime}\right)$ at an angle of incidence $\theta_{1}=60^{\circ}$ for two variants: 1) a hard prolate hemispheroid on a boundary with a hard medium; 2) a hard prolate

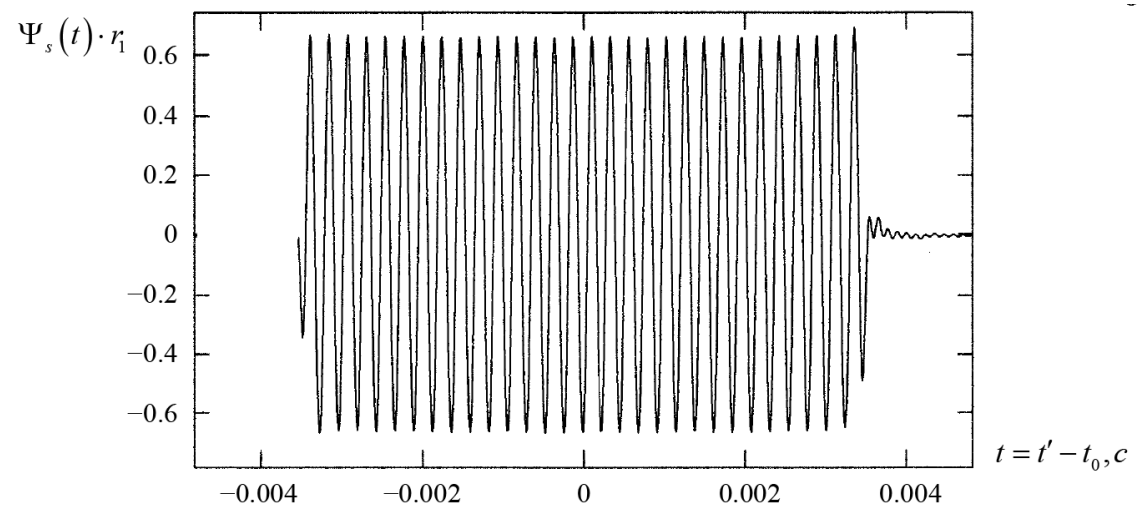

(a)

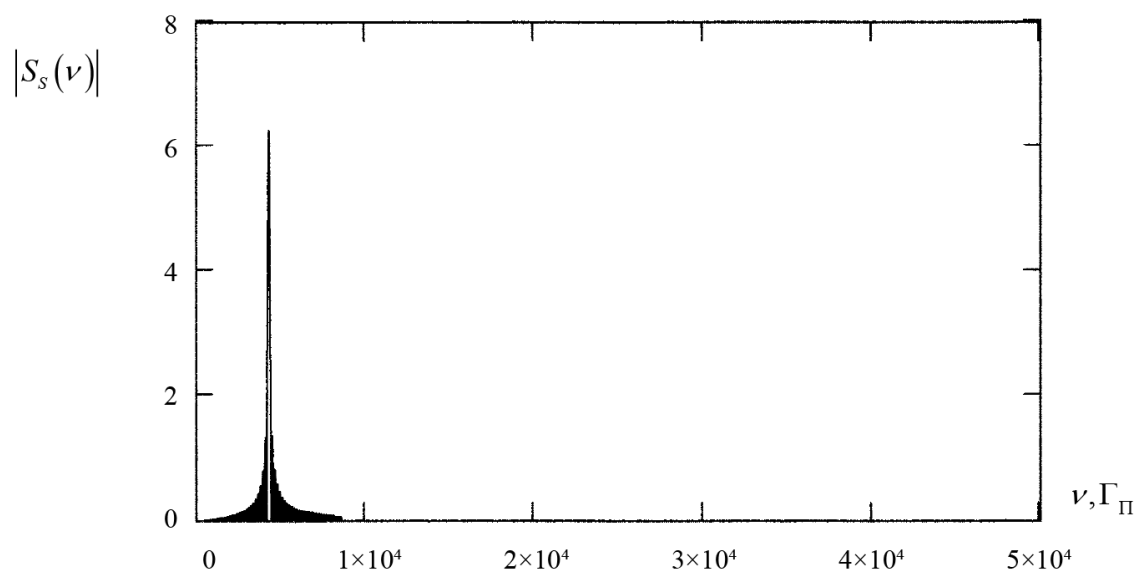

(b)

Figure 12. A mirror reflection pulse $r_{1} \cdot \Psi_{S}(t)$ (a); a normalized modulus of a spectrrum $\left|S_{S}(v)\right|$ of a pulse $r_{1} \cdot \Psi_{S}\left(t^{\prime}\right)(\mathrm{b})$. 


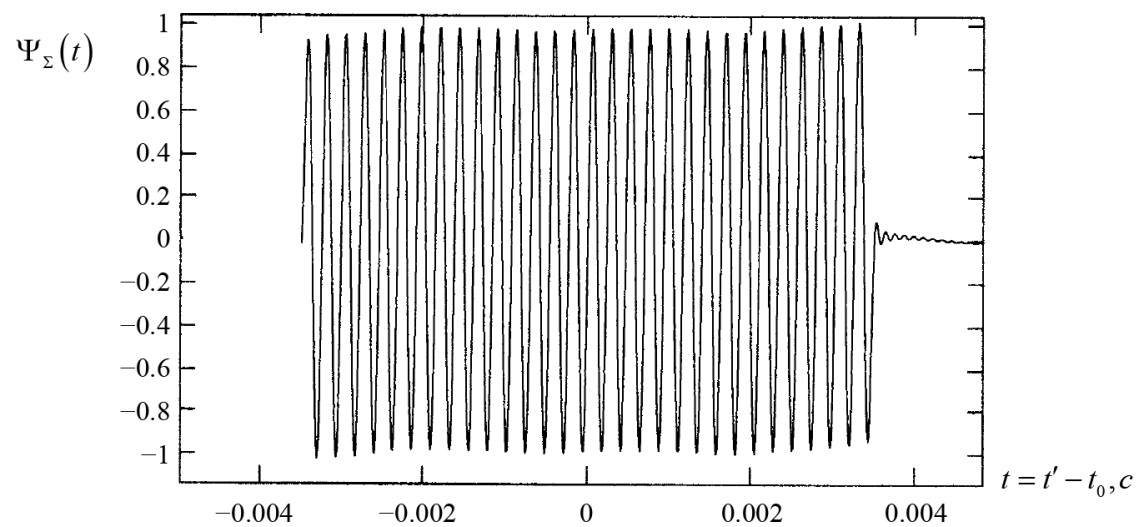

(a)

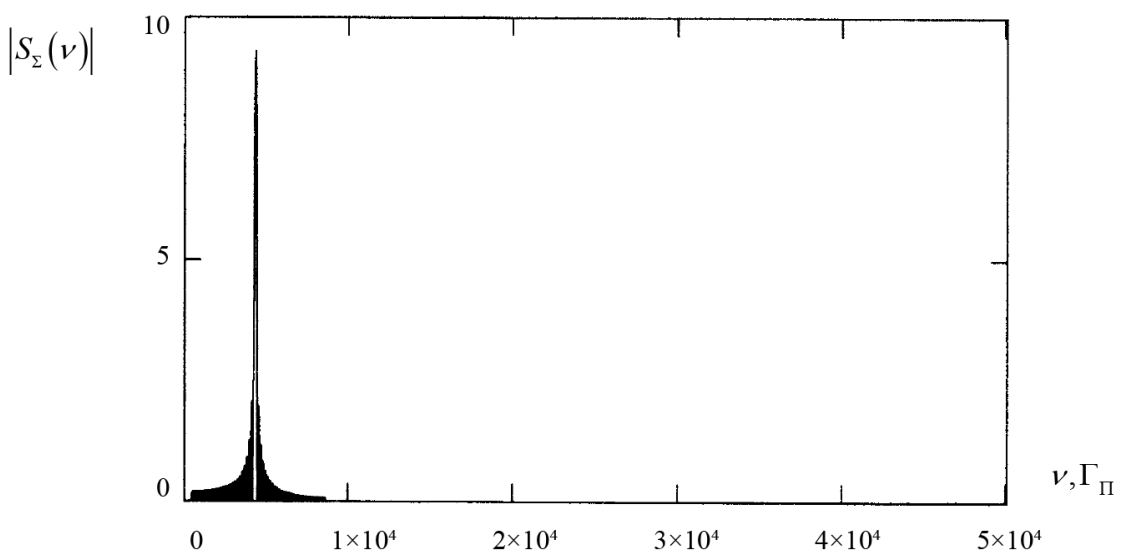

(b)

Figure 13. A diffracted pulse $\Psi_{\Sigma}(t)$ (a); a normalized modulus of a spectrum . $\left|S_{\Sigma}(v)\right|$ of a pulse $\Psi_{\Sigma}\left(t^{\prime}\right) \quad(\mathrm{b})$

hemispheroid on a boundary with a soft medium, In Figure 14 is presented a pulse $\Psi_{\Sigma}(t)$ (a) and a normalized modulus of a spectrum $\left|S_{\Sigma}(v)\right|$ for a first variant and in Figure 15 show same characteristics for a second variant

We see in Figure 16 a diffracted pulse $\Psi_{\Sigma}(t)$ and a modulus of its spectrum $\left|S_{\Sigma}(v)\right|$ in a direction of a mirror component for a hard oblate hemispheroid, located on a border of a liquid-an ideally soft mediumin such a way that its major axis lies in a plane of boundary. An irradiation angle remains a same. $\theta_{1}=30^{\circ}$.

In Figure 17 shows a diffracted pulse $\Psi_{\Sigma}(t)$ in a direction of a mirror component and a modulus of its spektrum $\left|S_{\Sigma}(v)\right|$ for a soft prolate hemispheroid placed on a border of a liquid-an ideally hard medium. Dimensions, orientation of a hemispheroid, a ratio of semi-axis in a same as in Figure 16. We note that for oblate and prolate hemispheroids located on a interface between media a diffracted pulse $\Psi_{\Sigma}(t)$ in a direction of a mirror component is obtained as a result of an interference of two reflected signals (from a foor of a hemispheroid and a boundary itself), herefore it is designated as a diffracted pulse/ $\Psi_{\Sigma}(t)$. 


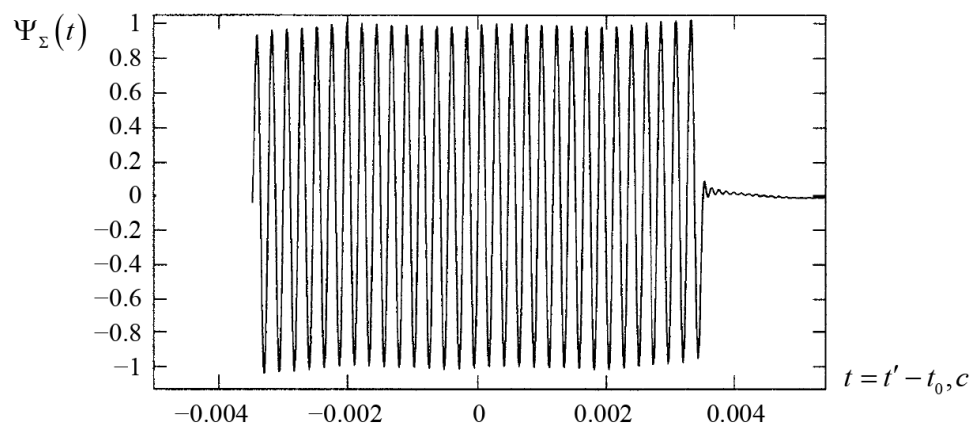

(a)

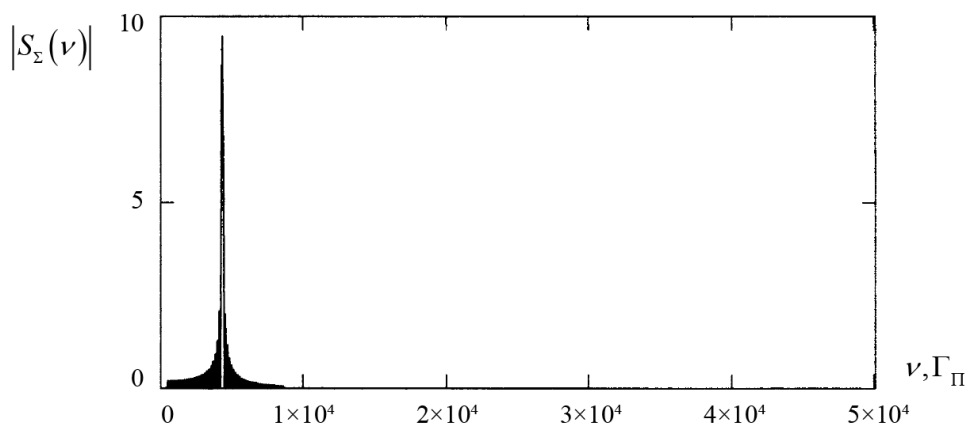

(b)

Figure 14. A mirror of reflection pulse $\Psi_{\Sigma}(t)$ for a hard prolate hemispheroid on a boundary with a hard medium (a); a normalized modulus of aspectrum $\left|S_{\Sigma}(v)\right|(\mathrm{b})$.

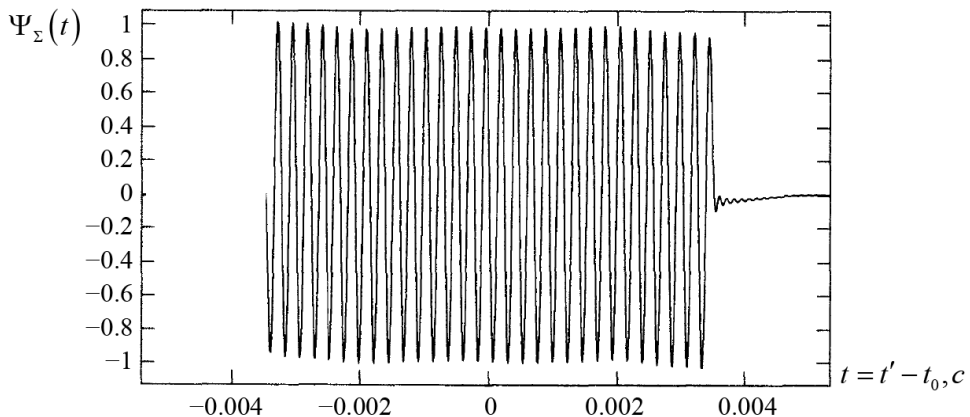

(a)

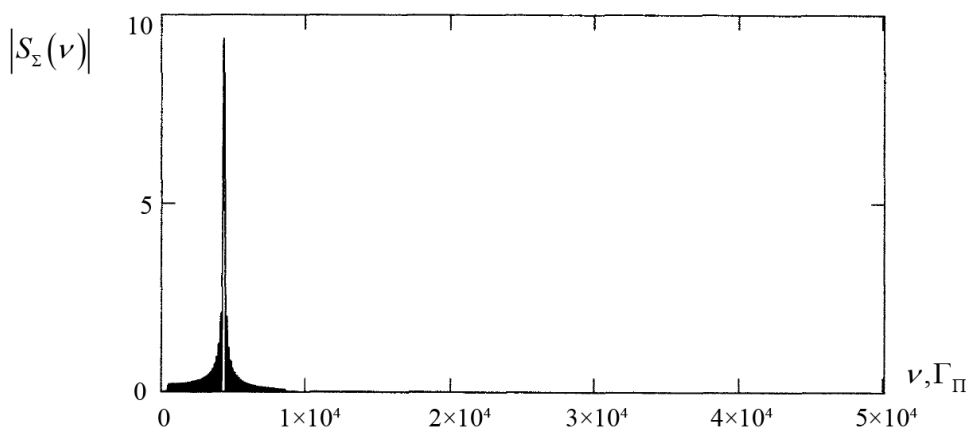

(b)

Figure 15. A mirror reflection pulse $\Psi_{\Sigma}(t)$ for a hard prolate hemispheroid on a boundary with a spft medium (a); a normalized modulus of a spectrum $\left|S_{\Sigma}(v)\right|(\mathrm{b})$. 


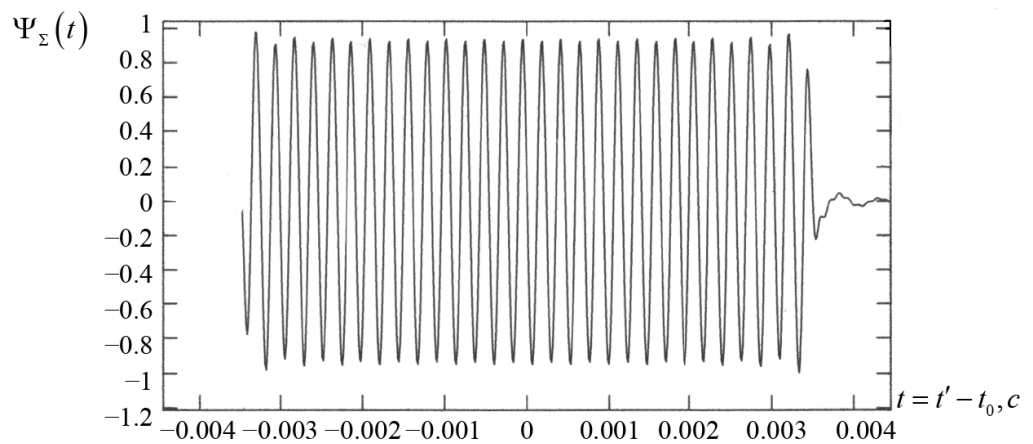

(a)

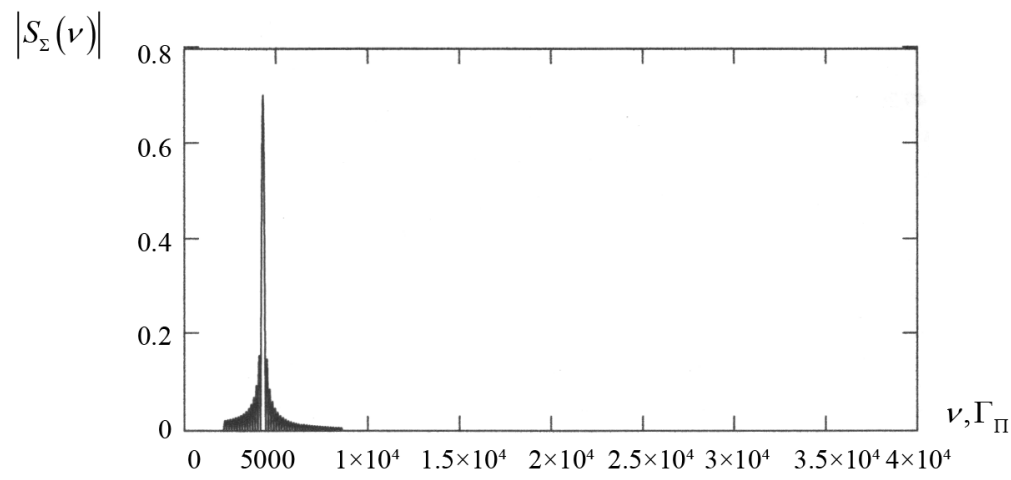

(b)

Figure 16. A mirror reflection pulse $\Psi_{\Sigma}(t)$ for a hard oblate hemispheroid (a); a normalized modulus of a spectrum $\left|S_{\Sigma}(v)\right|$ of a pulse $\Psi_{\Sigma}(t)$ (b).

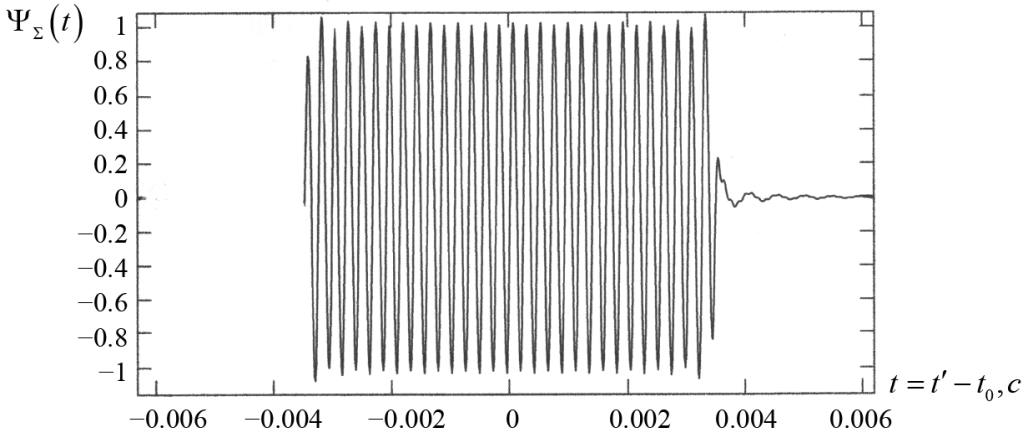

(a)

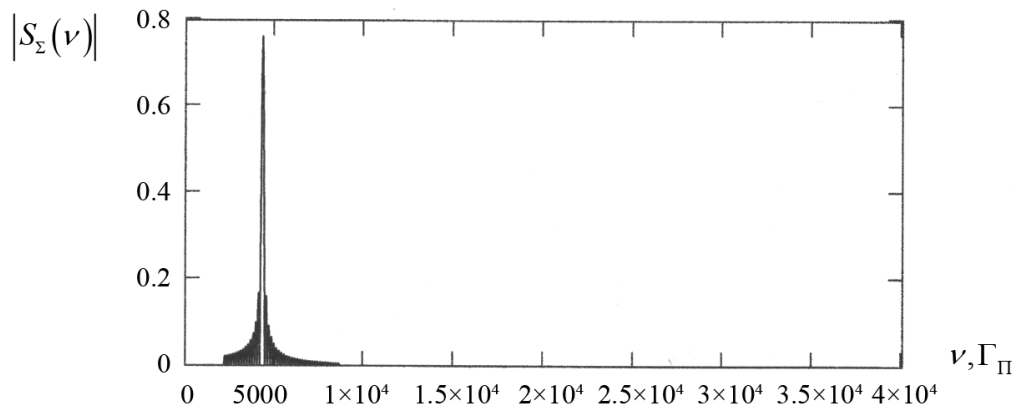

(b)

Figure 17. A mirror reflection pulse $\Psi_{\Sigma}(t)$ for a soft prolate hemispheroid (a); a normalized modulus of a spectrum $\left|S_{\Sigma}(v)\right|$ of a pulse $\Psi_{\Sigma}(t)$ (b). 
A picture of a reflection of sound by a spheroidal body located at a boundary of a liquid-an elastic bottom, we supplement it with angular scattering characteristics $R(\theta, \varphi)$ for a stationary sound signal with a fixed frequency. In Figure 18 shows a modulus $\left|R_{1}(\theta, \varphi)\right|$ of an angulare characteristic upon irradiation of a soft prolate spheroid with a plane wave at an angle $\theta_{1}=60^{\circ}$ and a modulus $\left|R_{\Sigma}(\theta, \varphi)\right|$ of an angulare characteristic upon irradiation with a plane wave reflected from a bottom.

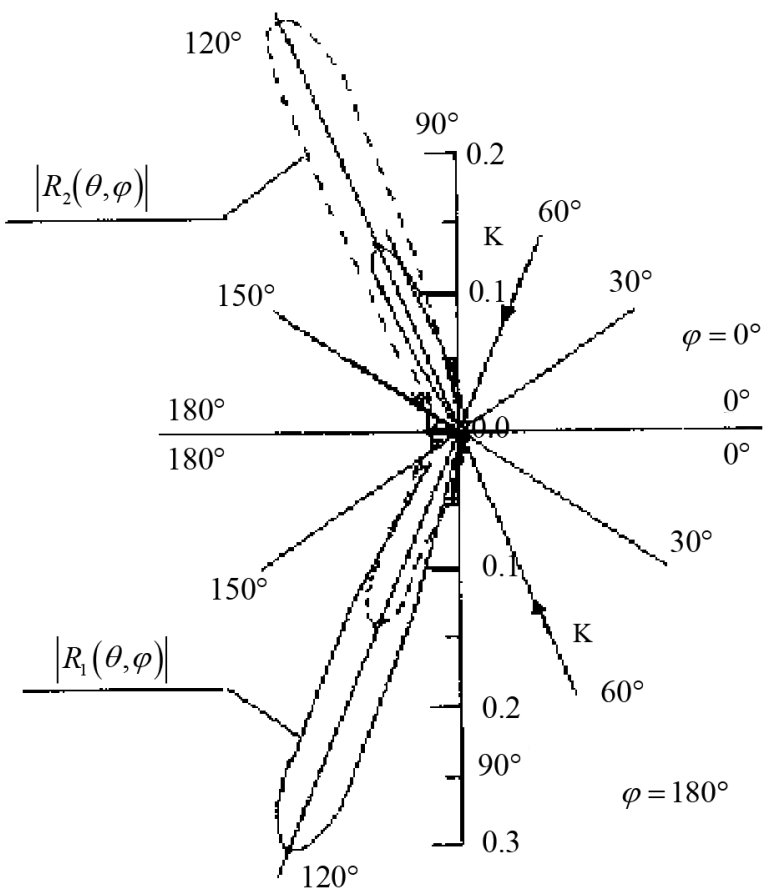

(a)

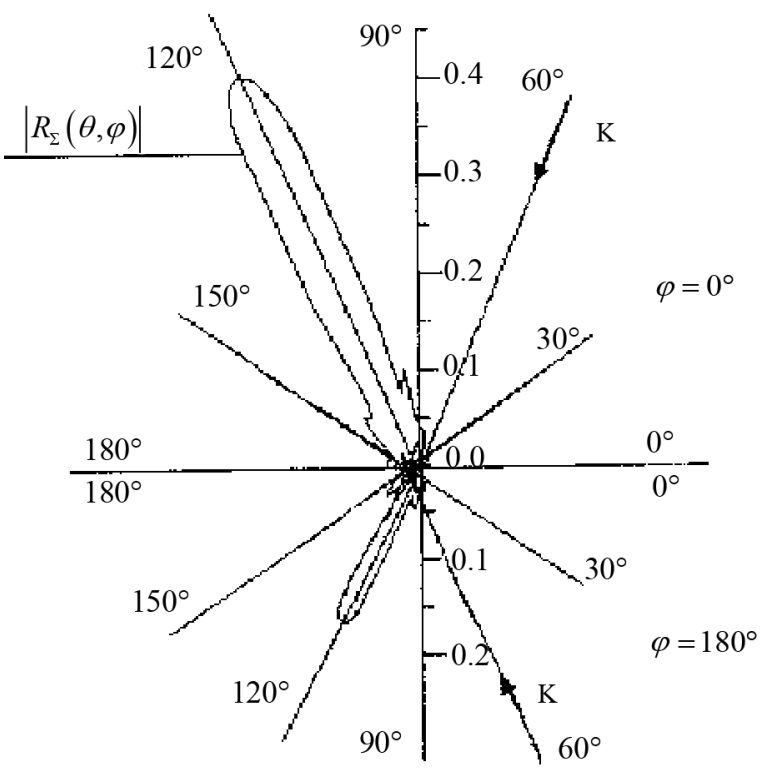

(b)

Figure 18. Modules of angular characteristics of a sound scattering by an ideal spheroid in free medium (a) and in a presence of a liquid-an elastic bottom boundary. 


\section{Ideal Spheroid in An Underwater Sound Channel}

A study of an effect of two boundaries on a field of a spheroidal scatterer will begin withan ideal spheroid placed in an underwater sound channel with non-reflecting boundaries [5] and irradiated with a pulsed signal with monochromatic filling [6]. At a depth $z_{0}$ of such a waveguide we place a point source of a rulsed signal and at a horizontal distance $r$ from it and at a depth $z_{2}-$ a spheroidal scatterer (Figure 19(a)). A velocity profile of sound in a symmetrical under-water sound channel is shown in Figure 19(b). A receiver of a scattered signal is compatible with a source, that is we will consider a combined system and find a spectrum of a scattered signal at this point. Since by a condition a velocity of sound depends only on a vertical coordinate $z$, in a horizontal plane rays are not bent and in each vertical half-plane, passing through a center of a scatterer a sound field will be independent of adjacent half-planes, and each half-plane there will be complex coefficients of excitation of a sound channel modes. A result, in each of these half-planes one observes an interference pattern of modes independent of neighboring half-planes. We are interested in a field in one vertical half-plane $P$ containing a coincident point of a source-receiver and a center of a scatterer. Since a scattered field in this half-plane does not depend on a behavior of this field in all other half-planes, we shall take it to be a same in all vertical yalf-planes and equal to a field in half-plane $P$. Approximately (without taking into account an effect of a medium on an angular scattering characteristic) a spectrum of a scattered signal at a location of a source will be equal to [6]:

$$
S_{2}(\omega)=\rho_{0}^{2}\left(r_{1}\right)^{-1} \sum_{m=1}^{M} \sum_{n=1}^{N_{m}} P_{m} P_{n, m} D_{n m}(\omega) \exp \left[-i\left(\kappa_{m} r_{1}+\kappa_{n m} r_{1}-\pi / 2\right)\right]
$$

where: $P_{m}=p_{m}\left(\rho_{0}\right)^{-1} \varphi_{m}\left(z_{2}\right) \varphi_{m}\left(z_{0}\right) ; p_{m}$-a mode excitation coefficient $m$; $\varphi_{m}\left(z_{2}\right)$-a intrinsic wave-guide function determined by boundary conditions;

(a)

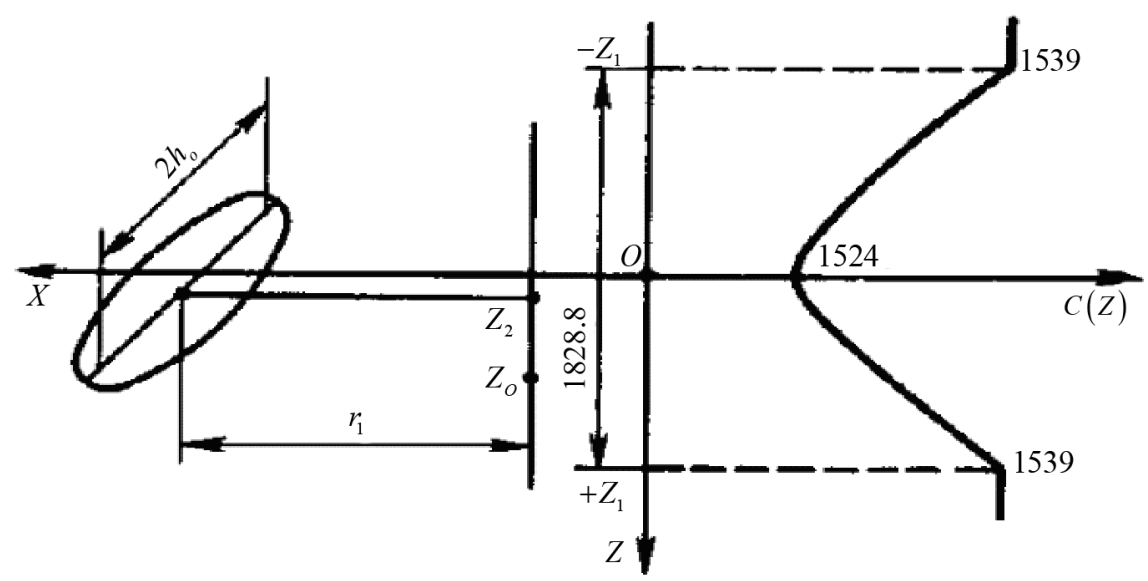

Figure 19. A mutual arrangement of a spheroidal scatterer and a source in a sound channel (a) and a profile of a velocity of sound in a sound channel with non-reflecting boundaries (b). 
$\rho_{0}$-density at source and receiver depth; $P_{n m}=\left(1 / \rho_{2}\right) \varphi_{n}\left(z_{2}\right) \varphi_{n}\left(z_{0}\right) ; \rho_{2}-$ density at a depth of a center of a scatterer; $D_{n m}(\omega)$-space-transfer function of body scattering for a $m$-th mode of a source and a $n$-th mode of a scatterer; $\kappa_{m}$ and $\kappa_{n m}$-horizontal components of wave numbers of modes of an incident and scattered waves res-pectively; $M$-a largest admissible source mode; $N$-a largest admissible scatterer mode for $m$-th mode of a source.

A dependence of a velocity of sound on a coordinate $z$ for a symmetric waveguide (see Figure 19(b)) has a form [5]:

$$
c(z)=(p|z|+q)^{1 / 2} .
$$

We will find a spectrum $S_{2}(\omega)$ at a combined point of a source and receiver for a ideal soft scatterer in a forn of a prolate spheroid with a coordinate of an outher surface $\xi_{0}=1.005$. We place a source (receiver) on an axis of a symmetric waveguide $\left(z_{2}=z_{0}=0\right)$, a major axis of a spheroid being perpendicular to an axis $Z$. An interfocus spheroid distance $2 h_{0}$ is assumed equal to $9.7 \mathrm{~m}$. A source generates a pulse signal with a duration $\tau_{0}=0.05 \mathrm{~s}$ at a frequency $f_{0}=400 \mathrm{~Hz} \quad(C=8.0)$. Space-transfer function $D_{n m}(\omega)$ is determined by a frequency and angular (in a plane $X O Z$ ) characteristics of a spheroid scattering. With a chosen velocity profile of a sound (see Figure 19(b)) a largest angle between a wave vectors in an incident and scattered waves is approximately $16^{\circ}$. Referring to an amplitude-phase angular characteristic of a scattering $D(\theta ; \varphi)$ of a soft spheroid in a plane $X O Z\left(\theta=90^{\circ}, \eta=0\right)$ (see Figure 20) we note that

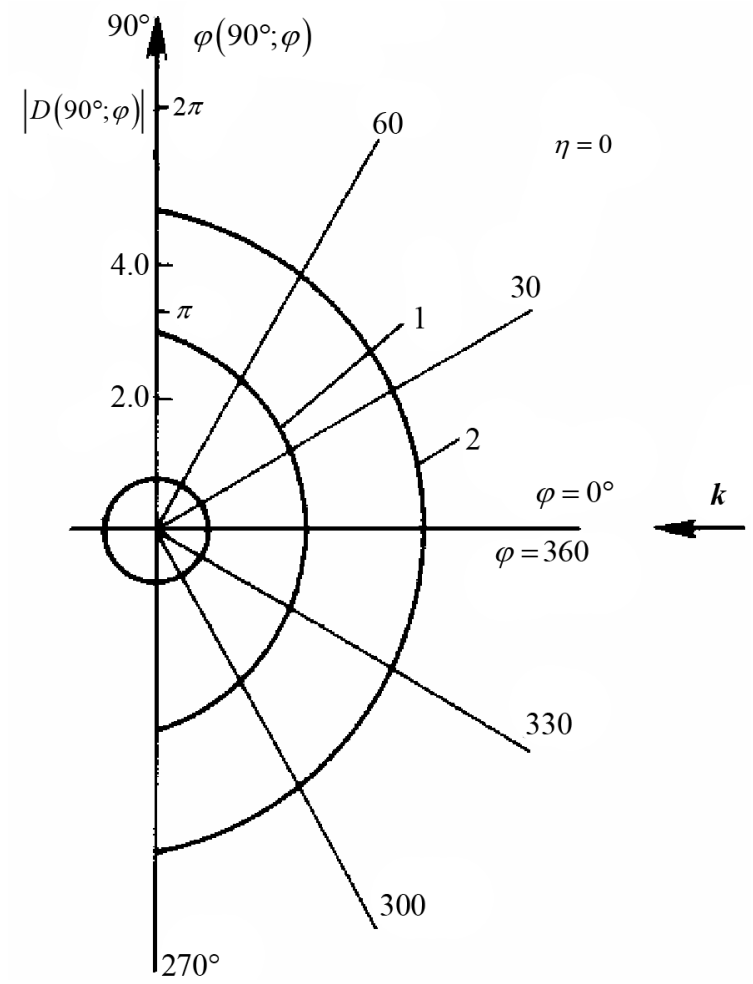

Figure 20. Amplitude-phase characteristic of sound scattering by a soft spheroid. 
even with a maximum wave size ( $C=10.0$ ) used in calculations $D\left(90^{\circ} ; \varphi\right)=\left|D\left(90^{\circ} ; \varphi\right)\right| \exp \left[i \psi\left(90^{\circ} ; \varphi\right)\right] \quad$ (an angular characteristic) is practically non-directional within angles $\phi=0 \div 16^{\circ}$ (see Figure 20). Such an approach, in which modes of an underwater sound channel are considered in a form of plane waves irradiating our spheroidal scatterer is also approximate. Curve 1 in Figure 20 refers to $6,\left|D\left(90^{\circ} ; \varphi\right)\right|$, curve 2 characterizes a phase $\psi\left(90^{\circ} ; \varphi\right)$ increased for all angles $\varphi$ by $\pi$. At lower wave dimensions characteristics will be even closer to circular. Therefore, taking into account a first assumption about uniform scattering in a horizontal plane, we can assume that each incident wave from a set of admissible modes will scatter a body uniformly in all directions (as non-directional scatterer) with an angular constant excitation coefficient $D(\omega)$ into which a shace-transfer function $D_{n m}(\omega)$. As a result, a spectrum of a scattered signal will be calculated by a formula [6]:

$$
S_{2}(\omega)=D(\omega) \rho_{0}^{2}\left(r_{1}\right)^{-1} \sum_{m=1}^{M} \sum_{n=1}^{M} P_{m} P_{n m} \exp \left[-i\left(\kappa_{m} r+\kappa_{n m} r-\pi / 2\right)\right] .
$$

\section{Diffraction Pulse Sound Signal on the Soft Prolate Spheroid Located in Plane Waveguide with the Hard Elastic Bottom}

We turn to a familiar problem of the diffraction of pulses on spheroial bodies I the plane waveguide [7] [8] [9] [10], retairing the upper boundary condition Dirichlet, waveguide dimensions and scatterer with respect to boundaries, replasing only ideal hard lower boundary on the elastic isotropic bottom. Physical parameters of the lower medium will correspond to $\phi$ the isotropic elastic bottom, but in their values, they will be very close to parameters of transversely-isotropic rock-a large gray siltstone [11]. The longitudinal wave velocity in this material is $4750 \mathrm{~m} / \mathrm{s}$, the transverse wave velocity-2811 m/s. When used in this case the method of imaginary sources need to enter the reflection coefficient $V$ for the each source [10], when displaying sources relative to the upper border sources, as before [7] [11] [12] [13] [14] [15], will change the sign on the opposite, which corresponds to a change of phase by $\pi$.

It is known to [10], that the imaginary sources method boundary conditions are not fulfilled strictly on any of borders of the waveguide even in the case of ideal boundary conditions of Dirichlet and Neumann. For the better fulfillment of these conditions in diffraction problems [7] [11] [12] [13] [14] [15] [16] were introduced imaginary scatterers by mirroring their relatively waveguide boundaries. Likewise conduct imaginary scatterers and in our problem and compare the sequence of reflected pulses [12] [13] [16] in the case of ideal borders and in presence of a hard elastic bottom in the waveguide. In [10] shows that the method of imaginary sources applicable in the case where the reflection coefficient $V$ will be a function of the angle of the incidence of the wave from a source relative to the normal to the boundary. In our case this angle will be determined by the mutual position of the source (real or imaginary) and the scatterers (real or imaginary), which falls the wave from the source. Since the receiver is combined 
with a real source $Q$, the sequence of reflected pulses will be determined by the quantity and amplitudes of reflected signals (from different scatterers) having the same propagation time from sources to scatterers and from scatterers to the point $Q$. Parameters of the waveguide, the position of the real source $Q$ (combined with the receiver) and the real scatterer remained unchanged compared [12] [13] [16]: $L=1000 \mathrm{~m}, H=400 \mathrm{~m}$, the real source $Q$ and real scatterer are located at the depth of $200 \mathrm{~m}$., the scatterer is the ideal soft prolate spheroid with the semi-axes ratio $a / b=10(a=0.279 \mathrm{~m})$ and its axis of a rotation is perpendicular to the plane of the figure (see Figure 21). The formula for the reflection coefficient $V_{0 N}$, where $N$-the number of a source, is given in [10]. For the calculatio9nof first five of reflected pulses us needed following reflection coefficients: $V_{03}$ in the direction of the first (real) scatterer 01, $V_{05}$ in the direction of the second (imaginary) scatterer $02, V_{06}$ in the direction of same the second scatterer.

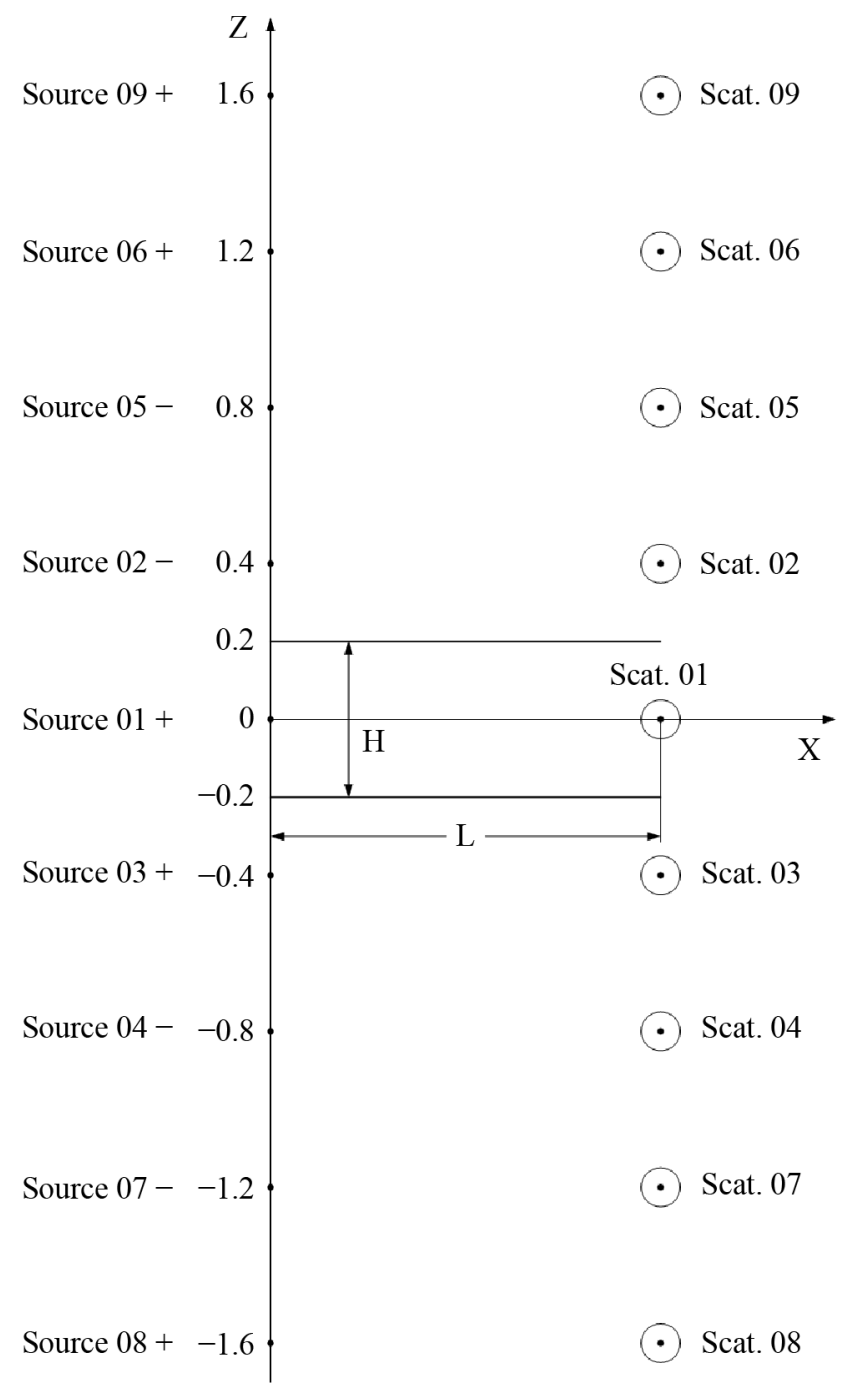

Figure 21. The mutual disposition of the pulse point-soirces and scatterers in the plane waveguide. 
As a result of simple calculations with the help of [7] obtain:

$V_{03}=0.9989+i 0.0633 ; \quad V_{05}=0.9989+i 0.0633 ; \quad V_{06}=0.6238+i 0.7897$. All three coefficients have turned complex, which mesns the total internal reflection at the boundary liquid-hard elastic bottom, therefore all three modules of reflection coefficients are equal 1.0 and real parts of first two coefficients are close to +1.0 , which is typical for the boundary liquid-absolutely hard bottom. The resulting sequence of calculations of first five reflected pulses is shown in Figure 22. We compare them to the sequence in Figure 23 for ideal boundaries [12] [13] [16]: $1^{\text {st }}$ and $4^{\text {th }}$ pulsews of Figure 22 are identical with first and second pulses of Figure 23, as for for $2^{\text {nd }}, 3^{\text {rd }}$ and $5^{\text {th }}$ pulses in Figure 22 in the case of ideal boundaries and symmetrical location of real a source and a scatterer relatively of boundaries of the waveguide, they are compensated each reflected pulses, i.e. $2^{\text {nd }}$, $3^{\text {rd }}$ and $5^{\text {th }}$ pulses (see Figure 22) show the difference in sequences of reflected pulses when replacing an absolutely hard bottom on an elastic hard bottom.

A similar pattern is observed for anisotropic bottom, such as silicon, in wich quasi-longitudinal wave velocity of about $8300 \mathrm{~m} / \mathrm{s}$ and quasi-transverse wave velocity of about $5700 \mathrm{~m} / \mathrm{s}$, with the secjnd quasi-transverse wave do not occur because of the problem statement [9]. Because of the high velocities of quasi-longitudinal and quasi-transverse waves total internal reflection effect at the anisotropic bottom manifest itself even more strongly than the isotrohic bottom.

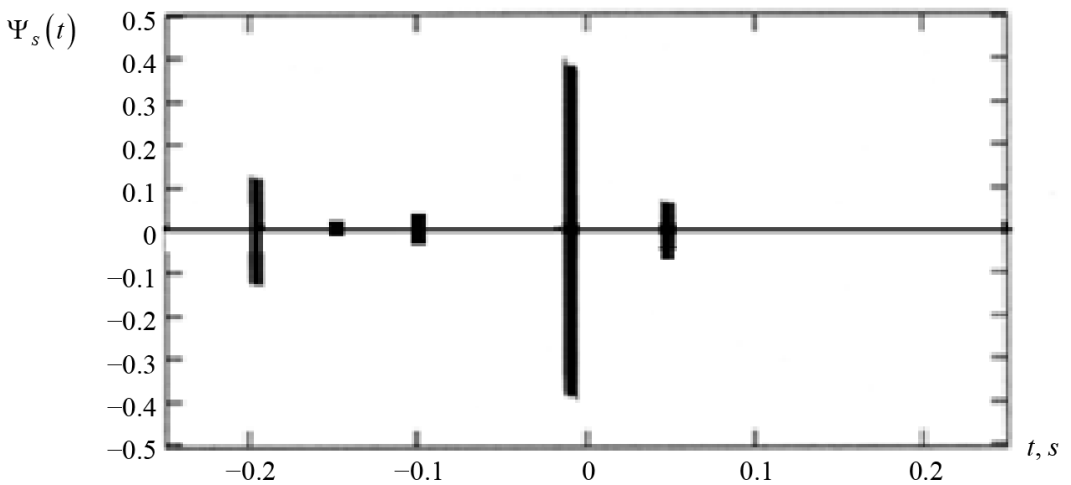

Figure 22. The normalized series of first five reflected impulses in the waveguide with the hard elastic bottom.

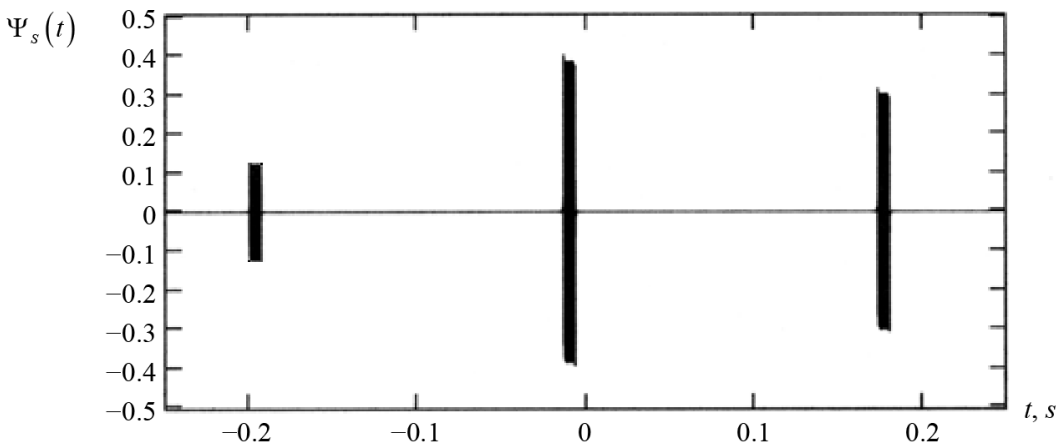

Figure 23. The normalized series of first three reflected impulses with the harmonic filling in the point $Q$. 


\section{Diffraction Pulse Sound Signal on Non-Analytical Elastic Scatterer Put in Plane Waveguide with Hard Elastic Bottom}

Based on the obtained solution, consider a more general problem of the diffraction of the pulsed sound signal on an elastic scatterer as a finite cylindrical shell, supplemented with two hemispherical shells (Figure 24) and placed in the waveguide with an elastic hard bottom (Figure 5), using the method of integral equations [16]-[21]. We note that a similar problem can be solved with the help of other methods: the boundary element method [20] [22]; T-matrix method [23]; the method of a potential [24]; the finite element method [25]; the method of Green's functions [26].

The first stage will solve the problem of the diffraction of a harmonic wave on a such shell. The density of the material of the shell is $\rho_{1}$, the Lame's coefficients $-\lambda$ and $\mu$. The shell was filled in the internal liquid medium with the density $\rho_{2}$ and the sound velocity $C_{3}$ and it was placad in the external liquid medium with the density $\rho_{0}$ and the sound velocity $C_{0}$. At the shell falls the plane harmonic wave with pressure $p_{i}$ under the angle $\Theta_{0}$ and with the wave vector $\boldsymbol{k}$.

As was shown in [16]-[21], the initial equation is integral equation, having the sense of the generalized Huygen's principle, for the displacement vector $\boldsymbol{u}(\boldsymbol{r})$ of the elastic shell:

$$
\boldsymbol{u}(\boldsymbol{r})=\iint_{S}\left\{\boldsymbol{t}\left(\boldsymbol{r}^{\prime}\right) G\left(\boldsymbol{r}^{\prime} ; \boldsymbol{r}\right)-\boldsymbol{u}\left(\boldsymbol{r}^{\prime}\right)\left[\hat{n}^{\prime} \Sigma\left(\boldsymbol{r}^{\prime} ; \boldsymbol{r}\right)\right]\right\} \mathrm{d} S\left(\boldsymbol{r}^{\prime}\right), \quad \boldsymbol{r} \in V,
$$

where $\boldsymbol{t}\left(\boldsymbol{r}^{\prime}\right)=\hat{n}^{\prime} T\left(\boldsymbol{r}^{\prime}\right)$ is the stress vector; $\hat{n}^{\prime} \equiv \hat{n}^{\prime}\left(\boldsymbol{r}^{\prime}\right)=\boldsymbol{n}^{\prime}\left(\boldsymbol{r}^{\prime}\right)$ is the single vector of the external along the relation to $S$ normal; $T\left(\boldsymbol{r}^{\prime}\right)$ is the stress tensor of the isotropic material; $G\left(\boldsymbol{r}^{\prime} ; \boldsymbol{r}\right)$ is the displacement Green's tensor; $\Sigma\left(\boldsymbol{r}^{\prime} ; \boldsymbol{r}\right)$ is the stress Green's tensor; if $\boldsymbol{r}$ concerns to the point of the surface $S$, in the left part of the Equation (19) will stand $\boldsymbol{u}\left(\boldsymbol{r}^{\prime}\right) / 2$.

The displacement vector $\boldsymbol{u}(\boldsymbol{r})$, the stress tensor $T(\boldsymbol{r})$, the displacement Green's tensor (Figure 25).

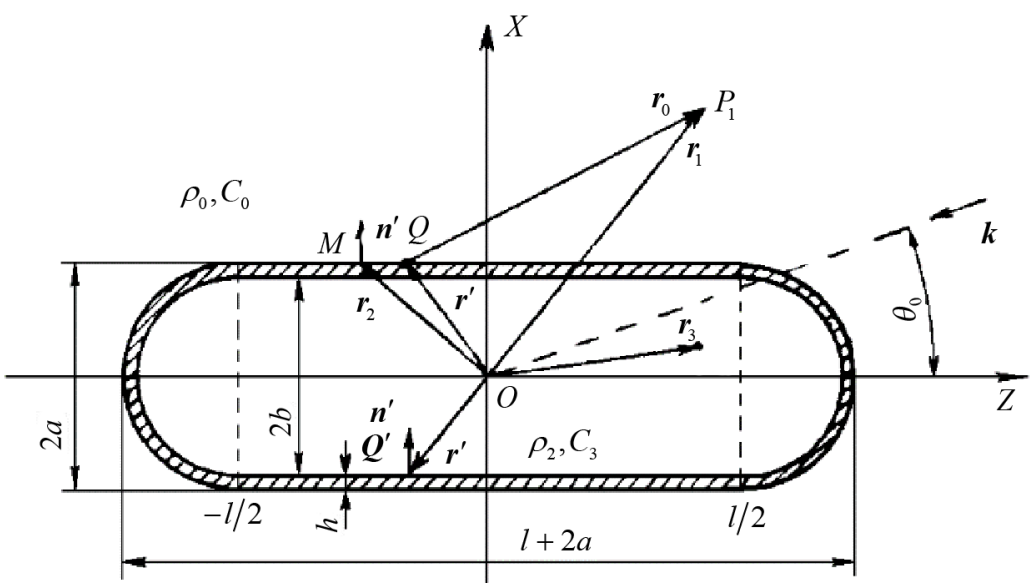

Figure 24. The elastic shell in the form of the terminal cylinder with the semi-spheres. 


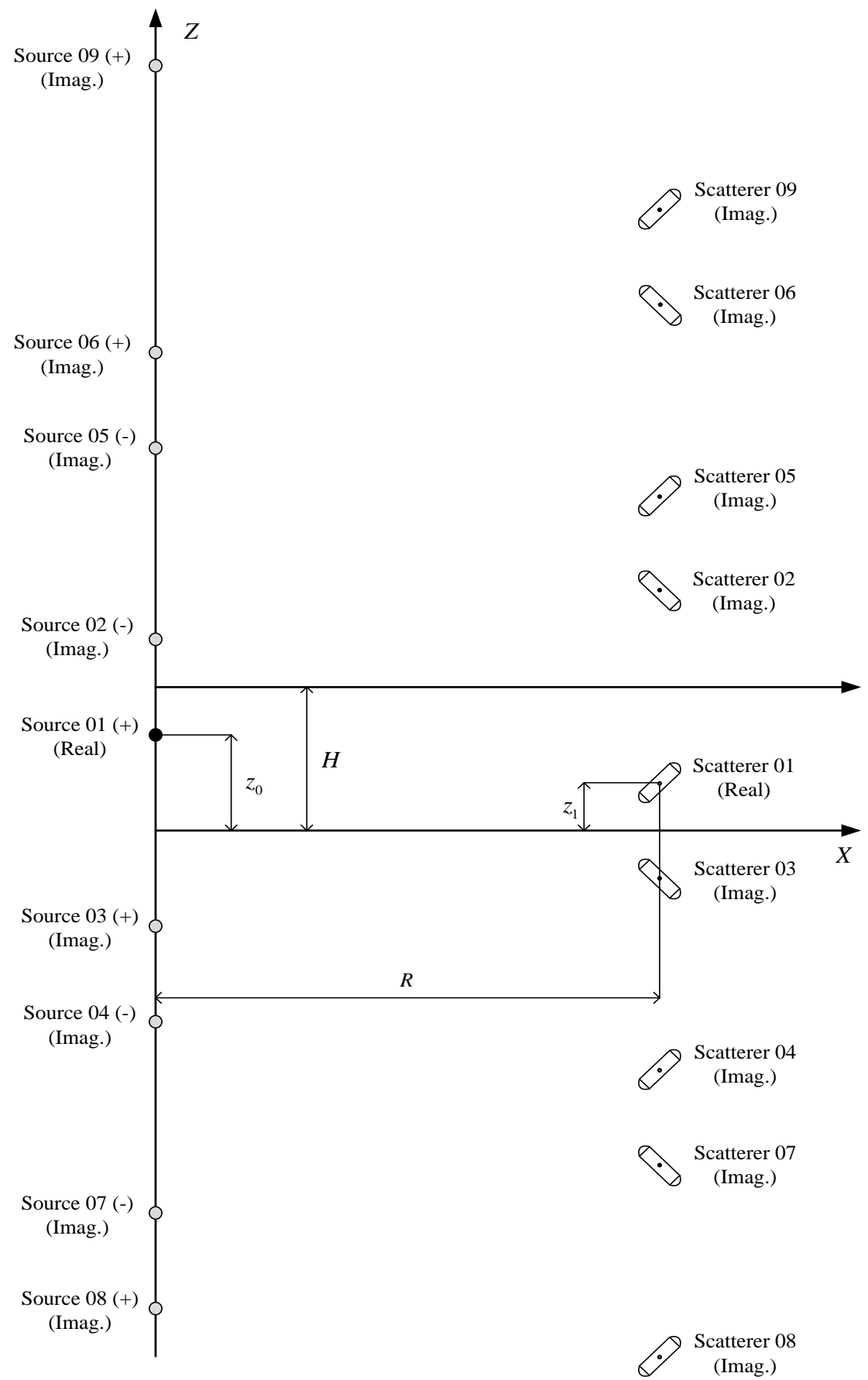

Figure 25. The mutual disposition of the impulse point-sources and scatterers in the plane waweguide.

The displacement vector $\boldsymbol{u}(\boldsymbol{r})$, the stress tensor $T(\boldsymbol{r})$, the displacement Green's tensor $G\left(\boldsymbol{r}^{\prime} ; \boldsymbol{r}\right)$ and the stress Green's tensor $\Sigma\left(\boldsymbol{r}^{\prime} ; \boldsymbol{r}\right)$ were connected between themselves by the following correlations [16] [21]:

$$
T(\boldsymbol{r})=\lambda I \nabla \boldsymbol{u}(\boldsymbol{r})+\mu(\nabla \boldsymbol{u}+\boldsymbol{u} \nabla),
$$

where $I=I_{L}+I_{T} ; \quad I_{L}=(\nabla \nabla) / \nabla^{2} ; \quad I_{L} \cdot I_{T}=0 ; \quad I_{T}=-\lceil\nabla(\nabla I)\rceil / \nabla^{2}, \quad I_{L} \quad$ and $I_{T}$ are the longitudinal and transverse single tensors for the Hamilton's opera- 
tor $\nabla$;

$$
\begin{aligned}
\sum\left(\boldsymbol{r}^{\prime} ; \boldsymbol{r}\right) & =\lambda I \nabla G\left(\boldsymbol{r}^{\prime} ; \boldsymbol{r}\right)+\mu\left[\nabla G\left(\boldsymbol{r}^{\prime} ; \boldsymbol{r}\right)+G\left(\boldsymbol{r}^{\prime} ; \boldsymbol{r}\right) \nabla\right] ; \\
G\left(\boldsymbol{r}^{\prime} ; \boldsymbol{r}\right)= & \left(1 / 4 \pi \rho_{t} \omega^{2}\right)\left\{k_{2} I g\left(k_{2}\left|\boldsymbol{r}^{\prime}-\boldsymbol{r}\right|\right)\right. \\
& \left.+\nabla^{\prime}\left[g\left(k_{1}\left|\boldsymbol{r}^{\prime}-\boldsymbol{r}\right|\right)-g\left(k_{2}\left|\boldsymbol{r}^{\prime}-\boldsymbol{r}\right|\right)\right] \nabla\right\},
\end{aligned}
$$

where $k_{1}$ and $k_{2}$ are the wave numbers of the longitudinal and transwerse waves in the material of the shell; $g\left(k_{2}\left|\boldsymbol{r}^{\prime}-\boldsymbol{r}\right|\right)=\exp \left(i k_{2}\left|\boldsymbol{r}^{\prime}-\boldsymbol{r}\right|\right) / 4 \pi\left|\boldsymbol{r}^{\prime}-\boldsymbol{r}\right|$ is the Green's function.

The second integral equation presents the Kirchhoff integral for the diffracted pressure $p_{\Sigma}\left(P_{1}\right)$ in the external medium:

$$
\begin{aligned}
C\left(P_{1}\right) p_{\Sigma}\left(P_{1}\right)= & -\iint_{S_{a}}\left\{p_{\Sigma}(Q)\left(\partial / \partial n^{\prime}\right)\left[\exp \left(i k r_{0} / r_{0}\right)\right]\right. \\
& \left.-\left[\exp \left(i k r_{0} / r_{0}\right)\right] \rho_{0} \omega^{2}\left(\mathbf{u n}^{\prime}\right)\right\} \mathrm{d} S_{a}+4 \pi p_{i}\left(P_{1}\right),
\end{aligned}
$$

where $p_{\Sigma}\left(P_{1}\right)=p_{i}\left(P_{1}\right)+p_{s}\left(P_{1}\right) ; p_{s}\left(P_{1}\right)$ is the scattered pressure in the point $P_{1} ; C\left(P_{1}\right)$ is the numerical coefficient, equal $2 \pi$, if $P_{1} \in S_{a}$ and $4 \pi$, if $P_{1}$ out $S_{a} ; S_{a}$ is the external surface of the shell; $Q$ is the point of the external surface of the shell.

For the pressure $p_{2}\left(M_{1}\right)$ in the internal liquid medium in the point $M_{1}$ is got the third integral equation:

$$
\begin{aligned}
C\left(M_{1}\right) p_{2}\left(M_{1}\right)= & \iint_{S_{b}}\left\{p_{2}\left(Q^{\prime}\right)\left(\partial / \partial n^{\prime}\right)\left[\exp \left(i k r_{3}\right) / r_{3}\right]\right. \\
& \left.-\left[\exp \left(i k r_{3}\right) / r_{3}\right] \rho_{0} \omega^{2}\left(\mathbf{u n}^{\prime}\right)\right\} \mathrm{d} S_{b},
\end{aligned}
$$

where $Q^{\prime}$ is the point of the internal surface of the shell;

$$
C\left(M_{1}\right)= \begin{cases}4 \pi, & \text { if } M_{1} \text { out } S_{b} ; \\ 2 \pi, & \text { if } M_{1} \in S_{b} ;\end{cases}
$$

$S_{b}$ is the internal surface of the shell.

To the integral Equations (19), (23) and (24) are added the boundary conditions on the external $\left(S_{a}\right)$ and internal $\left(S_{b}\right)$ surfaces of the shell:

1) at the both surfaces of the shell the tangent stresses are equally null:

$$
\left.\tau_{i}\right|_{S_{a}}=0 ;\left.\tau_{i}\right|_{S_{b}}=0 ; i=1,2 ;
$$

2) the normal stress $\sigma_{n^{\prime}}$ at the external surface of the shell is equally the diffracted pressure $p_{\Sigma}$, but at the internal surface is equally the pressure $p_{2}$

$$
\left.\sigma_{n^{\prime}}\right|_{S_{a}}=p_{\Sigma} ;\left.\quad \sigma_{n^{\prime}}\right|_{S_{b}}=p_{2} ;
$$

In the conformity with the conditions (7) and (8) the stress vector $\boldsymbol{t}\left(\boldsymbol{r}^{\prime}\right)$ in the Equation (1) is equal:

$$
\boldsymbol{t}\left(\boldsymbol{r}^{\prime}\right)=\left.p_{\Sigma} \boldsymbol{n}^{\prime}\right|_{S_{a}} ; \boldsymbol{t}\left(\boldsymbol{r}^{\prime}\right)=\left.p_{2} \boldsymbol{n}^{\prime}\right|_{S_{b}} ;
$$

3) the continuity of the normal component of the displacement at the both boundaries of the shell: 


$$
\left.\begin{array}{l}
u_{n^{\prime}}=\left.\left(1 / \rho_{0} \omega^{2}\right)\left(\partial p_{\Sigma} / \partial n^{\prime}\right)\right|_{S_{a}} ; \\
u_{n^{\prime}}=\left.\left(1 / \rho_{2} \omega^{2}\right)\left(\partial p_{2} / \partial n^{\prime}\right)\right|_{S_{b}} \cdot
\end{array}\right\}
$$

The substitution of integral Equations (19), (23) and (24) in the boundary conditions gives the system of equations in terms of unknown functions $p_{\Sigma} p_{2}$ and the components of the displacement vector $\boldsymbol{u}$ at the both surfaces of the shell. To obtain numerical solution of this system the integral equations are replaced the quadrature formulas and the grid of the nodal points is chosen at both surfaces of the shell as well as it has be done for the ideal non-analytical scatterers [16] [17].

For choosing boundary conditions we will have the integrals of the two types: the integrals with the isolated special point and the integrals which are considered of the sence of the principal meaning. The method of the calculation of the second types was described in [16].

Thus calculated reflection characteristics of the harmonic signal with frequency $v$ can determine the spectral reflectance function $S_{S}(2 \pi v)$ and it can help be applying a Fourier transform we obtain a temporary function of the reflected pulse $\Psi_{S}\left(t^{\prime}\right)$ [27]:

$$
\Psi_{S}\left(t^{\prime}\right)=\frac{1}{\pi} \operatorname{Re} \int_{0}^{\infty} S_{S}(2 \pi v) \mathrm{e}^{+i 2 \pi v t} \mathrm{~d}(2 \pi v)
$$

Similarly using spectral reflectance characteristics of elastic bodies of spheroidal form [16] [28] [29] [30] [31], we can compute sequences of reflected pulses in the waveguide with hard elastic bottom and for their.

\section{Conclusions}

In the first part of the review we investigate an interaction of a scatterer and an interface between media; it is shown that a main role in this is played by interference effects. The second part of the review is devoted to a study of a spektrum of a scattered field of an ideal prolate spheroid placed in an underwater sound channel with non-reflecting boundaries. The third part of the review demonstrates the effect of a bottom structure on a series of pulses, reflected from a spheroidal body located in a plane waveguide.

As a result of the research we can draw three conclusions:

1) During studying propagation and diffraction of pulse signals in a plane waveguide, one needs to use the method of imaginary sources as pulses like bundles of energy spread to any directions (including and along the axis of the waveguide) with the group velocity which does not exceed the sound velocity, namely the group velocity based the method of imaginary sources;

2) Replacing the hard elastic bottom on the absolutely hard bottom is acceptable to those sources (real and imaginary) from which waves in the fall to the hard elastic bottom try total internal reflection.

3) We have adopted the model of image sources and image scatterer is gute 
acceptable (due to internal reflection), at least, for first five calculated reflected pulses in a plane wave-guide with hard elastic bottom.

\section{References}

[1] Kleshchev, A.A. (1977) Scattering of Sound by Spheroidal Bodies Located at Interface between Media. Soviet Physics. Acoustics, 23, 404-410.

[2] Sommerfeld, A. (1950) Differential Equations in Partial Derivatives of Physics. Foreign Literature, M., 456 p.

[3] Ivanov, E.A. (1968) Diffraction of Electromagnetic Waves on Two Bodies. Science and Technology, Minsk, $583 \mathrm{p}$.

[4] Kleshchev, A.A. (1979) Scattering of Sound by Spheroidal Body Located at Interface between Media. Soviet Physics. Acoustics, 25, 143-145.

[5] Tolstoy, I. and Kley, K.S. (1969) Acoustic of Ocean. World, M., 304 p.

[6] Kleshchev, A.A. and Klyukin, I.I. (1974) The Spectral Characteristics of the Scattering of the Sound by Body, Placed in the Sound Channel. Soviet Physics. Acoustics, 20, 470-473.

[7] Kleshchev, A.A. (2015) Diffraction of Pulse Sound Signals on Elastic Bodies of Spheroidal Form Put in Plane Waveguide. MIT, Vol. 2, 77-81.

[8] Kleshchev, A.A. (2017) Analytical and Non-Analytical Scatterers on Plane Waveguide with Hard Elastic Bottom, Irradiated by Pulse Sound Signal. American Journal of Modern Physics, 6, 51-56.

[9] Lekhnitskiei, S.G. (1977) Theory of Elasticity of Anisotropic Elastic Body. Science, M., $416 \mathrm{p}$.

[10] Brechovakikh, L.M. (1957) Waves in Laminated Mediums. Publ. Acad. Scien. SSSR, M., 502 p.

[11] Kleshchev, A.A. (2015) Pulse Sound Signals Diffraction on Elastic Bodies of Analytical and Non-Analytical Forms, Put in Plane Waveguide. Zeitschrift fur Naturforschung $A, 70,419-427$.

[12] Kleshchev, A.A. and Kuznetsova, E.I. (2011) Scattering of Pulse Sound Signals by the Spheroidal Body, Put in Plane Wavegui de. Coll. Proc. Russ. Acoust. Soc. XXIV Session. GEOS, M., Vol. 1, 743-745.

[13] Kleshchev, A.A. and Kuznetsova, E.I. (2012) Diffraction of Impulse Signals on Spheroidal Body, Put in Plane Waveguide. International Journal of Theoretical and Mathematical Physics, 2, 211-214.

[14] Kleshchev, A.A. (2013) Diffraction of Pulse Sound Signals on Elastic Spheroidal Shell, Put in Plane Waveguide. Advanced Studies in Theorrsetical Physics, 7, 697-705.

[15] Kleshchev, A.A. (2014) Diffraction of Sound Signals at Elastic Shell of Non-Analytical Form Put in Plane Waveguide. Advances in Signal Processing, 2, 46-49.

[16] Kleshchev, A.A. (2012) Hydroacoustic Scatterers. Prima, S.-Pb., 268 p.

[17] Kleshchev, A.A. (1989) Scattering of Sound by Ideal Bodies of Non-Analytical Form. Tr. Lenin. Korablestr. Inst. Generalship. Syst., 95-99.

[18] Kleshchev, A.A. (1993) Method of Integral Equations in Problem of Sound Diffraction on Elastic Shell of Non-Analytical Form. Journal of Technical Acoustics, 2, 65-66. 
[19] Kleshchev, A.A. (2013) Method of Integral Equations in Problem of Sound Diffraction on Bodies of Non-analytical Form. Marine Messenger, 2, 94-98.

[20] Seybert, A.F., Wu, T.W. and Wu, X.F. (1988) Radiation and Scattering of Acoustic Waves from Elastic Solids and Shells using the Boundary Element Method. The Journal of the Acoustical Society of America, 84, 1906-1912.

[21] Podstrigach, J.S. and Poddubnjak (1986) Scattering of Sound Beams of Spherical and Cylindrical Form. Naukova Dumka, Kiev, 264 p.

[22] Brebbia, C.A. and Walker, S. (1982) Boundary Element Techniques in Engineering. World, M., $242 \mathrm{p}$.

[23] Peterson, B. and Strom, S. (1975) Matrix Formulation of Acoustic Scattering from Multilayered Scatterers. Journal of the American Statistical Association, 57, 2-13.

[24] Kupradze, V.D. (1963) Methods of Potential in Theory of Elasticity. Fizmatgiz, M., $472 \mathrm{p}$.

[25] Dushin, A.Y., Il'menkov, S.L., Kleshchev, A.A. and Postnov, V.A. (1989) Use of Finite El Sement Method to Solution of Problems of Sound Radiating by Elastic Shells. Proc. All-Union Symp. Interaction of Acoustical Waves with Elastic Bodies, Tallinn, 89-91.

[26] Il'menkov, S.L., Kleshchev, A.A. and Klimenkov, A.S. (2014) The Green's Function Method in the Problem of Sound Diffraction by an Elastic Shell of Noncanonical Shape. Acoustical Physics, 60, 579-586.

[27] Kharcevich, A.A. (1957) Spectrum and Analisis. GITTL, M., 236 p.

[28] Kleshchev, A.A. (1986) Three-Dimensional and Two-Dimensional (Axis-Symmetrical Characteristics of Elastic Spheroidal Scatterers. Akusticheskii Zhurnal, 32, 268-270.

[29] Kleshchev, A.A. and Klyukin, I.I. (1987) Principles of Hydroacoustics. Shipbuilding, L., $224 \mathrm{p}$.

[30] Kleshchev, A.A. (2012) Debye and Debye-Type Potentials in Diffraction, Radiation and Elastic Wave Propagation Problems. Acoustical Physics, 58, 338-341.

[31] Kleshchev, A.A. (2014) Resonance Scattering of Sound by Spheroidal Elastic Bodies and Shells. Acoustical Physics, 60, 253-261. 